\title{
The market for scoops: a dynamic approach
}

\author{
Ascensión Andina-Díaz ${ }^{1}$ (D) José A. García-Martínez ${ }^{2}$ - Antonio Parravano ${ }^{3}$
}

Received: 6 August 2018 / Accepted: 27 March 2019 / Published online: 6 April 2019

(c) The Author(s) 2019

\begin{abstract}
We present a dynamic model of competition in the media industry in which behavioral media outlets compete for the publication of scoops, and both the publication of scoops and their veracity determine an outlet's future audience. We study the dynamics of the audiences of outlets and how those dynamics relate to two issues: how harsh a society is with the publication of false stories, and how similar media outlets are in their editorial standards for quality. For the case of a duopoly, we show that there is only one stable stationary state. In that equilibrium, the two outlets coexist and which one leads the market (the low-standard outlet or the high-standard outlet) depends on a combination of the two issues mentioned above. We then use numerical simulations to analyze the general case with more than two outlets. The numerical results corroborate most of the analytical insights gained for the duopoly case. We also use numerical simulations to draw up predictions on the number of outlets that will survive in the industry in the long run.
\end{abstract}

Keywords Media industry · Competition - Editorial standard - Stochastic dynamics . Deterministic dynamics

\footnotetext{
We thank participants at the 2018 Economics of Media Bias Workshop in Cologne and the 2018 JEI Conference in Barcelona for useful comments. We also thank the Editor Juan D. Moreno-Ternero and two referees for their insightful comments. We acknowledge financial support from the Ministerio de Economía y Competitividad through projects MTM2014-54199-P and ECO2014-52345-P, and the Junta de Andalucía through project SEJ2011-8065. Antonio Parravano also acknowledges the hospitality of the University of Málaga, where part of this research was carried out. The usual disclaimer applies.
}

$凶$ Ascensión Andina-Díaz aandina@uma.es

José A. García-Martínez jose.garciam@umh.es

Antonio Parravano parravan3@gmail.com

1 Dpto. Teoría e Historia Económica, Universidad de Málaga, Málaga, Spain

2 Dpto. Estudios Económicos y Financieros, Universidad Miguel Hernández, Elche, Spain

3 Centro de Física Fundamental, Universidad de Los Andes, Mérida, Venezuela 
JEL Classification D25 - L10 $\cdot$ L82

\section{Introduction}

Casual evidence suggests important differences in the quality of journalism from one country to another. The traditional press is one example to look at. Among other possible differences, a striking one is the coexistence of countries where high-quality newspapers have large readerships with others where high-circulation newspapers have surprisingly low editorial standards. The cases of the USA and Spain, where quantity aspects (newspaper circulation) and quality aspects (accuracy of information and trustworthiness) go hand in hand, are thus in sharp contrast to the case of the UK, where the newspapers that lead the market in terms of circulation, The Sun and The Daily Mail, are far down the ranking in terms of trust and accuracy of news. That ranking is led by The Times and The Guardian. ${ }^{1}$

Differences in quantity and quality go beyond country-specific issues and can be found when different media sectors from the same region are compared. An example is the proliferation of fake news, a phenomenon that affects many spheres and sectors of the media industry but is especially severe and important on social media and online information platforms. How can these differences be explained? What determines which media outlets are more successful in a society?

This paper proposes a model of competition for audience that serves to draw predictions as to the fundamental aspects that determine what type of media outlets lead a market. In our opinion, this question requires a dynamic approach, because the accuracy of a story printed today may well affect an outlet's future audience. Our objective is to determine what ingredients can play a role in explaining the differences observed in the real world. We acknowledge that the problem is complex and that many variables may be involved. For example, issues such as press penetration in different social classes, ideological considerations, and prices, which are not considered in this paper, may have major effects. In this sense, the present paper should be seen as an attempt to identify some initial general properties of the problem.

Our contribution is to pin down the importance of two variables in explaining the dynamics: On the one hand, the consumers' discomfort with fake news, i.e., how harsh consumers are with the publication of false stories, and on the other hand the nature of competition between media outlets, i.e., whether they are homogeneous in their editorial standards for quality and thus compete for the same stories, or differ and offer different products.

In this paper, we use two approaches to characterize the dynamics of the market. On the one hand, we use a mean-field approach to find analytical solutions for the long-term average state of a duopoly media market. On the other hand, we numeri-

\footnotetext{
${ }^{1}$ For Spain, see the "Digital News Report 2017," by Reuters Institute, which identifies El País and El Mundo as the leading newspapers (in terms of both circulation and trustworthiness). For the USA, see Mitchell and Weisel (2014) and Agility PR, according to which USA Today, Wall Street Journal and New York Times are the leading newspapers (again, in terms of both circulation and trustworthiness). For the UK, see Newman (2017) and PRWeek/OnePoll.
} 
cally follow the stochastic dynamics of a market with several media outlets. ${ }^{2}$ These approaches are not standard in the literature of media economics. In this respect, our simple model could serve as a guideline for future analyses of other, more complex economic systems.

The model is as follows. We consider a media industry with $N$ non-strategic media outlets and a finite but large enough time horizon. At each step in time, referred to as a day, one scoop is released. We assume that each scoop can be published by only one media outlet. Media outlets compete for scoops according to a rule that determines the probability of an outlet receiving a scoop at a given step in time. The rule establishes that this probability is an increasing function of the outlet's audience on that particular day. ${ }^{3}$ Scoops contain information on a relevant variable and the information contained can be ex-ante more or less accurate. We refer to the ex-ante accuracy of the information as the quality of the scoop. We assume that the quality is i.i.d. across periods and that each scoop is either true or false with a probability proportional to its quality. Media outlets are characterized by their own editorial standards for quality, which establish the minimum quality that each outlet requires from a scoop in order to publish it. We consider that the editorial standards of outlets are exogenously given and invariant throughout the dynamics. We further consider that an outlet's editorial standard determines the behavior of that outlet in that it prescribes that the outlet will publish the scoop when it is of sufficiently high quality and will reject it otherwise. ${ }^{4}$ A scoop that is published is referred to as a story. After publication, consumers learn whether the story is true or false. If no story is published, consumers learn nothing. The behavior of an outlet at a time step, namely whether it publishes a story or not and whether the story is true or false, affects that outlet's future audience and hence its probability of receiving the next day's scoop.

We analyze the dynamics of the audiences of the outlets and the frequency of scoop publication (referred to as their share of the news). Our objective is to understand the effect on the dynamics of two issues: The valuation of news by consumers and the market dispersion. To that end, we take two complementary approaches: first we propose a mean-field analysis that substitutes the stochastic dynamics by a deterministic one. ${ }^{5}$ In particular, at each time step we substitute the random assignation of a scoop to an outlet by its expected value. This simplification enables us to obtain analytical

\footnotetext{
2 We point out that there are many open questions related to the dynamics of the dissemination of information in a society. Most of these dynamic systems cannot be fully examined in analytical terms. In such cases, numerical simulations are the only reliable alternative way to study their dynamics.

3 The formulation of this rule is supported by anecdotal evidence, such as the Lewinsky and bin Laden's death stories. In these examples, the scoops were first received by renowned media outlets (Newsweek and The New Yorker, respectively), and it was only after these outlets refused to publish them that the stories were passed on to less influential outlets (Drudge Report and The London Review of Books, respectively). See Andina-Díaz and García-Martínez (2018) for a detailed discussion of both stories.

4 In Appendix A, we discuss a one-shot game with rational agents that micro-founds this behavioral assumption.

5 Mean-field analysis is an approach commonly used in statistical physics and epidemiology that approximates the dynamics of a system by its expected motion. In economics, it was introduced only at the turn of this century, but in the last ten years its use has increased rapidly. We refer readers to the pioneering study by López-Pintado (2006, 2008) and Jackson and Rogers (2007) that use the mean-field approach to study the diffusion of a certain behavior in a social network. More recent examples of the use of this technique in economic literature are Lelarge (2012) and Kreindler and Young (2013).
} 
results for the case of two media outlets. Second, we perform numerical simulations on the stochastic dynamics and obtain predictions for the general case with more than two outlets. ${ }^{6}$

Our analytical results for the mean-field approach with two outlets show that there are two stationary states: one in which the two outlets coexist, i.e., they both receive positive audiences and shares of the news, and one in which only the outlet with the lower editorial standard is active in the market, i.e., the high-standard outlet ends up with zero audience and zero share of the news. We also study the stability of these two solutions and find that the stationary state in which the outlets coexist is the only stable one. The results that follow therefore focus on this last case. We then study what market features determine which outlet receives a higher audience and/or a higher share of the news. We show that whether it is the low-standard outlet or the high-standard outlet depends on a combination of the two issues described above. In particular, we obtain that when the punishment for publishing false stories is not very high the low-standard outlet always has more audience. In this case, that outlet also has the higher share of the news. In this sense, our results predict that societies that are especially generous with outlets that break news and do not penalize them when their stories are shown to be false will have media industries dominated by outlets with low editorial standards. However, when the cost of publishing a false story is sufficiently high and the outlets are similar enough in their editorial standards, the high-standard outlet may lead the market (in terms of both audience and share of the news). It requires the media outlets to be sufficiently homogeneous. Otherwise, the high-standard outlet may be the leader in audience but the low-standard one will always have a higher share of the news.

Regarding the stochastic dynamics, the results of the numerical simulations show that most of the insights gained in the mean-field approach with two outlets are robust to the consideration of more outlets. We also use numerical simulations to obtain predictions that cannot be inferred from the mean-field approach, such as predictions as to how many outlets will survive in the industry in the long run and their characteristics. We find that the harsher consumers are with the publication of false stories and/or the more similar the editorial standards of the media outlets are, the more outlets will survive in the long run. By contrast, when consumers like consuming news and do not care whether printed stories are true or false and/or when outlets are very different in their vetting processes for stories, the results suggest that only a small number of outlets endure in the long run.

This work is related to the literature on the economics of the media, an extensive area of literature that has grown rapidly in the last twenty years. The first papers in this literature were mostly interested in analyzing the effects of the media on economic policies and outcomes. See Besley and Burgess (2001), Djankov et al. (2003), Strömberg (2004b). A second generation of papers studied whether news provision was biased (Groseclose and Milyo 2005; Egorov et al. 2009 and Larcinese et al. 2011) and what the determinants of the biases were (Strömberg 2004a; Mullainathan and Shleifer 2005; Gentzkow and Shapiro 2006; Baron 2006; Petrova 2008; Ellman and Germano

\footnotetext{
6 The use of numerical simulations is also becoming increasingly common in economic literature. Examples are Harrington (1999), Vega-Redondo et al. (2005), Chen and Huang (2008), Casari (2008) and Arifovic et al. (2015), all with a micro-focus. In macroeconomic analysis, its use is very common, e.g., in the analysis of dynamic stochastic general equilibrium models.
} 
2009). At the same time, a different group of researchers focused their attention on the study of the media as a two-sided market. See Rochet and Tirole (2003), Anderson (2006), Doyle (2013). Currently, research on the economics of the media is extremely varied, but there is a tendency toward analyzing online news markets (Yang and Chyi 2011; Halberstam and Knight 2016; Germano and Sobbrio 2017) and their effects on political outcomes (Allcott and Gentzkow 2017; Boxell et al. 2017; Campante et al. 2018), collective action (Acemoglu et al. 2017; Little 2016; Enikolopov et al. 2016, 2017) and more generally, social outcomes (Gentzkow and Shapiro 2011; Quattrociocchhi et al. 2014; Bakshy et al. 2015).

Our contribution to this literature is to present a new approach to the study of competition and audience in the media industry. Previous research shows that competition can reduce the quality of news (Zaller 1999; Cagé 2014), can induce outlets to ideologically differentiate from competitors (Gentzkow et al. 2014) and can lead them to bias the news in an attempt to product-differentiate (Anand et al. 2007). It has also been shown that competition can reduce the possibility of media capture by the government (Besley and Prat 2006), increase media self-censorship on issues sensitive to advertisers (Germano and Meier 2013), and increase giving and volunteering (Adena 2016). In contrast to previous research, our interest lies not in the effects of competition but in its determinants, from a long-run perspective. To the best of our knowledge, this is new in the literature.

The rest of the paper is organized as follows. In Sect. 2, we propose a stochastic dynamic model of competition and audience in the media industry consisting of $N$ recursive equations that account for changes in audience in a market with $N$ outlets. In Sect. 3, we substitute the stochastic dynamics by a deterministic dynamics and analyze the resulting mean-field model for the case of two media outlets. This simplification enables us to obtain analytical solutions for the audiences of outlets and study the stability of the stationary states. In Sect. 4, we perform numerical simulations to study the stochastic dynamics with two, five, and ten media outlets. Finally, Sect. 5 concludes. The paper also contains Appendices, which are organized as follows. In Appendix A, we propose and analyze a one-shot model with rational agents that helps us show the rationale behind the behavioral assumptions that we make in Sect. 2. In Appendix B, we present results which are complementary to the stochastic dynamics analyzed in Sect. 4. Finally, Appendix C contains the proofs.

\section{The model}

We consider a market for news with $N$ media outlets and a mass of consumers. All the agents in the model, i.e., media outlets and consumers, are non-strategic and take decisions according to behavioral rules. Appendix A presents a model with rational agents that micro-founds the behavioral assumptions made in this section.

Each $t \in T=\{1,2, \ldots\}$ represents a time step which, without loss of generality, we identify with a day. For the sake of simplicity, two states of the world are assumed. At each time $t$, the state of the world is $\omega(t) \in \Omega$, with $\Omega$ representing the set of states. We assume that $\omega$ is distributed according to a known distribution function. At each time $t$, there is a binary signal $s(t)$ on the state of the world. We refer to the signal $s(t)$ 
as the scoop at time $t$. Let $\mu$ be the probability that a scoop is true, i.e., the quality of the scoop. We consider $\mu$ to be a random variable distributed according to a uniform distribution function in $[0,1]$. The realization of the random variable at $t$ is denoted by $\mu(t)$, where $\mu(t)=P(s(t)=j \mid \omega(t)=j)$.

Each media outlet $i \in\{1,2, \ldots, N\}$ is characterized by a threshold $\mu_{i} \in[0,1]$, which sets a lower bound on the quality of a scoop required by outlet $i$ in order to publish it. We refer to $\mu_{i}$ as the editorial standard of outlet $i$. Without loss of generality, we order media outlets according to their editorial standards, so that $0 \leq \mu_{1}<\mu_{2}<$ $\ldots<\mu_{N} \leq 1$ and assume that an outlet's editorial standard is invariant over time. This is a reasonable assumption if it is considered that an outlet's editorial standard and editorial line constitute a kind of moral or ethical charter that a media outlet abides by once they have been adopted.

Conditionally on an outlet having received a scoop, threshold $\mu_{i}$ determines whether outlet $i$ publishes it or not. More precisely, we consider that if $\mu(t)>\mu_{i}$ outlet $i$ publishes the scoop and suppresses it otherwise. The optimality of this decision rule is analyzed in Appendix A. We denote by $a_{i}(t) \in\{0,1\}$ the action of outlet $i$ at time $t$, where $a_{i}(t)=1$ when the outlet reports the scoop and $a_{i}(t)=0$ otherwise. A scoop that is printed/published is referred to as a story.

We assume that at every $t$, if a story is published consumers immediately learn the value of the random variable $\omega(t)$ with probability 1 , whereas if no story is published there is no learning. ${ }^{7}$ Thus, after a report, consumers always receive information that is valuable for assessing the accuracy of a story, which enables them to reward/punish a media outlet according to its behavior and the consumers' valuation of the news. In this respect, we consider that consumers reward the publication of a story and punish the publication of a false story. The model in Appendix A proposes a rationale for this behavior. For the sake of simplicity, let $\alpha \in(0,1)$ be the punishment imposed by the consumers on an outlet that publishes a false story and let $1-\alpha$ be the reward for an outlet that obtains a story and publishes it. Thus, $\alpha$ measures the relative weight of the punishment. Hence, the higher $\alpha$ is, the greater the discomfort of consumers with fake news is, then the harsher the punishment by consumers for the publication of false stories (in relative terms). ${ }^{8}$

The reward/punishment may be expected to have a positive/negative impact on an outlet's reputation, market share, and/or audience. Although the intensity of the impact may differ from one of these points to another, it is reasonable to consider that they will all move in the same direction. For the sake of specificity, we focus our attention here on the evolution of one of these values: The audiences of outlets. Let $A_{i}(t)$ denote the audience of media outlet $i$ at date $t$, and let $\widetilde{A}_{i}(t)=\frac{A_{i}(t)}{\sum_{j=1}^{N} A_{j}(t)} \in[0,1]$,

\footnotetext{
7 A more natural assumption would be to consider that consumers do not immediately learn the accuracy of a story but rather takes a number of periods to do so. Based on numerical simulations showing that the results do not change with the introduction of a delay, we have stuck to the simplest formulation.

8 The reward to an outlet for publishing a scoop is not necessarily negatively correlated with the punishment parameter $\alpha$. In fact, consumers might be both avid for stories and strongly concerned about false news, i.e., they may give high rewards and punishments. In order to reduce the number of parameters in the model, we assume that the reward is given by $1-\alpha$ and focus on the relative weight of these two concepts. This enables us to compare the numerical results that we obtain in Sect. 4 with the relatively simple analytical solutions that we get in Sect. 3 and thus show how our approach to the market dynamics works.
} 
with $\sum_{i=1}^{N} \widetilde{A}_{i}(t)=1$, be outlet $i$ 's normalized audience. With a focus on normalized audiences, note that $\widetilde{A}_{i}(t+1)$ should belong to the interval $\left[\widetilde{A}_{i}(t), 1\right]$ after outlet $i$ publishes a story. This is so when $\widetilde{A}_{i}(t+1)=\widetilde{A}_{i}(t)+(1-\alpha)\left(1-\widetilde{A}_{i}(t)\right)$. This illustrates the reward that an outlet receives for publishing a story. However, if the story is false, consumers impose a punishment on the outlet that reduces its audience by $\alpha \widetilde{A}_{i}(t)$. Putting the reward and the punishment together, we have the following recursive equation:

$$
A_{i}(t+1)=\widetilde{A}_{i}(t)+\Phi\left[(1-\alpha)\left(1-\widetilde{A}_{i}(t)\right) I_{a}-\alpha \widetilde{A}_{i}(t) I_{s \neq \omega}\right]
$$

where $\Phi>0$ is a parameter that modulates the change in an outlet's audience due to the publication of a single scoop, and $I_{a}$ and $I_{s \neq \omega}$ are the following indicator functions: ${ }^{9}$

$$
I_{a}=\left\{\begin{array}{l}
1 \text { if } a_{i}(t)=1, \\
0 \text { otherwise, }
\end{array} \text { and } I_{s \neq \omega}=\left\{\begin{array}{l}
1 \text { if } a_{i}(t)=1 \text { and } s(t) \neq \omega(t), \\
0 \text { otherwise }
\end{array}\right.\right.
$$

Finally, the dynamic equation can be written either in terms of the audience [Eq. (2)] or the normalized audience [Eq. (3)], which are equivalent. For simplicity, in the analysis we use Eq. (2).

$$
\begin{aligned}
A_{i}(t+1)= & \frac{A_{i}(t)}{\sum_{j=1}^{N} A_{j}(t)} \\
& +\Phi\left[(1-\alpha)\left(1-\frac{A_{i}(t)}{\sum_{j=1}^{N} A_{j}(t)}\right) I_{a}-\alpha \frac{A_{i}(t)}{\sum_{j=1}^{N} A_{j}(t)} I_{s \neq \omega}\right], \\
\widetilde{A}_{i}(t+1)= & \frac{\widetilde{A}_{i}(t)+\Phi\left[(1-\alpha)\left(1-\widetilde{A}_{i}(t)\right) I_{a}-\alpha \widetilde{A}_{i}(t) I_{s \neq \omega}\right]}{\sum_{j=1}^{N}\left(\widetilde{A}_{j}(t)+\Phi\left[(1-\alpha)\left(1-\widetilde{A}_{j}(t)\right) I_{a}-\alpha \widetilde{A}_{j}(t) I_{s \neq \omega}\right]\right)} .
\end{aligned}
$$

Note that the rule above considers that variations in an outlet's audience are proportional to the outlet's previous normalized audience $\widetilde{A}(t)$. In particular, it implies that the higher an outlet's audience at $t$, the smaller the gain from publishing a scoop and the higher the cost of being shown to have printed a false story. On the other hand, the smaller an outlet's audience, the greater the gain from breaking news and the smaller the cost of publishing a false story. We consider this property to give an accurate picture of the functioning of the real-world media industry.

We assume that an outlet's audience at $t$ affects the probability of it receiving a scoop at that time step. The idea is to model a situation in which outlets with higher audiences are more able to capture scoops than smaller ones. This is a reasonable assumption if we consider that outlets with larger market shares have larger newsrooms and are thus more likely to uncover events. Let $p_{i}(\mu(t))$ denote the probability of outlet $i$ receiving a scoop of quality $\mu(t)$ at time $t$. Remember that there is one scoop per day and that only one media outlet can publish it. We assume that probability $p_{i}(\mu(t))$ is given by:

\footnotetext{
9 The effect of parameter $\Phi$ on the results is discussed in Appendix B. If we were to consider that different scoops have different impacts on a society, we could assume that at each time step $t$ the scoop released has an impact $\Phi_{t}$ that is a random variable from a known distribution function.
} 


$$
p_{i}(\mu(t))=\frac{\theta_{i}(t) A_{i}(t)}{\sum_{k=1}^{N} \theta_{k}(t) A_{k}(t)},
$$

where

$$
\theta_{i}(t)= \begin{cases}1 & \text { if } \mu_{i} \leq \mu(t) \\ 0 & \text { if } \mu_{i}>\mu(t)\end{cases}
$$

Note that the process above determines that given a scoop of quality $\mu(t)$, only media outlets with editorial standards lower than $\mu(t)$ are active recipients of the scoop, i.e., have a positive probability of receiving it. Note also that because the publication strategy of any outlet, say $i$, is to publish a scoop whenever $\mu(t)>\mu_{i}$, the specification above determines that any media outlet that receives a scoop publishes it. This simple modeling approach should be seen as a reduced form of a more general mechanism consisting of the following: (i) At all $t$, any outlet has a positive probability of receiving the scoop; (ii) the probability of an outlet receiving a scoop is the same as its normalized audience; (iii) on receiving a scoop, the outlet decides whether to publish it or not; and (iv) if the outlet chooses to publish it, the mechanism at $t$ stops; however, if the outlet rejects it, the process of assigning the scoop starts again, with the outlet that rejected the scoop being taken out of the pool and the rest of the outlets now having probabilities of receiving the scoop that are equal to each outlet's new normalized audience. ${ }^{10}$ Note that the outcome of this more general mechanism is equivalent to that under the simpler form, where scoops are offered directly to those outlets that are willing to publish them. For the sake of simplicity, we stick to the proposed formulation.

Finally, note also that the above process determines a division of the scoop's quality space $[0,1]$ into different intervals, according to which competition for a scoop is softer or tougher. In particular, ceteris paribus $N$, the lower the quality $\mu(t)$, the softer the competition, as only outlets with low editorial standards are willing to publish it. When $\mu(t)<\mu_{1}$, no outlet wants to publish the scoop. On the other hand, the higher $\mu(t)$, the tougher the competition, as more outlets are willing to publish it. Note also that the degree of competition for scoops depends on how similar or different the editorial standards of the media outlets are. In particular, the more similar the editorial standards are, the tougher the competition is. In contrast, the more different the media outlets are, the softer the competition in the market for scoops is.

The model described considers a stochastic sequence of scoops and $N$ media outlets that repeatedly interact over time, with interactions determining the dynamics of the outlets' audiences. This process defines a system of $N$ stochastic recursive equations, which is analyzed in Sect. 4. Prior to this, in Sect. 3 we study a mean-field approach to the problem that allows us to study the average dynamics, characterize the stationary states, study the stability and perform a comparative static exercise for the case of an industry with two media outlets.

\footnotetext{
10 This mechanism finds support in the real-world examples of the Lewinsky scandal and the story of bin Laden's death. See footnote 3.
} 


\section{The mean-field dynamics}

This section considers a mean-field approach to the problem. It makes the following simplification: We substitute the stochastic dynamics described in Eq. (2) by a deterministic dynamics. In particular, for every $t$ and outlet $i$, we substitute the random variables $I_{a}$ and $I_{s \neq w}$ by their expected values $F_{i \text {,news }}$ and $F_{i \text {,false: }}$ :

$$
\begin{aligned}
A_{i}(t+1)= & \frac{A_{i}(t)}{\sum_{j=1}^{N} A_{j}(t)}+\left[(1-\alpha)\left(1-\frac{A_{i}(t)}{\sum_{j=1}^{N} A_{j}(t)}\right) F_{i, \text { news }}(t)\right. \\
& \left.-\alpha \frac{A_{i}(t)}{\sum_{j=1}^{N} A_{j}(t)} F_{i, \text { false }}(t)\right] .
\end{aligned}
$$

Note that the dynamics above makes $\Phi=1$. This is without loss of generality. ${ }^{11}$

Since the quality of a scoop $\mu(t)$ is assumed to be a random variable uniformly distributed in $[0,1]$, the expected value of the random variable $I_{a}$ is:

$$
\begin{aligned}
F_{i, \text { news }}(t)= & \int_{\mu_{i}}^{\mu_{i+1}} p_{i, i}(t) \mathrm{d} \mu \\
& +\int_{\mu_{i+1}}^{\mu_{i+2}} p_{i, i+1}(t) \mathrm{d} \mu+\cdots+\int_{\mu_{N-1}}^{\mu_{N}} p_{i, N-1}(t) \mathrm{d} \mu+\int_{\mu_{N}}^{1} p_{i, N}(t) \mathrm{d} \mu,
\end{aligned}
$$

where $p_{i, k}(t)=\frac{A_{i}(t)}{\sum_{l=1}^{k} A_{l}(t)}$ is the probability of outlet $i$ receiving a scoop of quality $\mu_{k} \leq \mu \leq \mu_{k+1}$ at time $t$. Note that $F_{i \text {, news }}$ represents the fraction of news published by $i$ in stationary conditions (out of the total number of news items received by the outlets). Note also that $\sum_{i=1}^{N} F_{i \text {,news }}(t)=1-\mu_{1}$, since a fraction $\mu_{1}$ of stories does not meet the minimum quality for publication by any outlet.

Let $\mu_{N+1}=1$. This fraction can thus be rewritten as: ${ }^{12}$

$$
F_{i, \text { news }}(t)=\sum_{k=i}^{N} \int_{\mu_{k}}^{\mu_{k+1}} p_{i, k}(t) \mathrm{d} \mu .
$$

Additionally, since the probability of a scoop being true is proportional to its quality $\mu$, the expected value $F_{i \text {,true }}(t)$ of outlet $i$ publishing a true story at $t$ is:

$$
F_{i, \text { true }}(t)=\sum_{k=i}^{N} \int_{\mu_{k}}^{\mu_{k+1}} \mu p_{i, k}(t) \mathrm{d} \mu .
$$

\footnotetext{
11 As observed in Appendix B, the stationary state solution does not depend on $\Phi$, which only affects the amplitude of the fluctuations around the solution. In particular, the higher $\Phi$ is, the greater the fluctuations are. Hence, by considering $\Phi=1$ we analyze the stability of the system under adverse conditions.

12 Note that given (4), the expression in (7) can also be written as $F_{i, \text { news }}(t)=\int_{\mu_{i}}^{1} p_{i}(\mu(t)) \mathrm{d} \mu$.
} 
Therefore,

$$
F_{i, \text { false }}(t)=F_{i, \text { news }}(t)-F_{i, \text { true }}(t)
$$

is the expected value of the random variable $I_{s \neq w}$ and represents the fraction of the stories published by $i$ at $t$ that are false.

\subsection{Two outlets}

Next we restrict the analysis to the case of two media outlets. This simplification allows us to obtain analytical results and identify some general properties of the system that we subsequently test with numerical simulations in the stochastic dynamics.

Let 1 and 2 denote the two media outlets, and consider $\mu_{1}<\mu_{2}$. In the following, we refer to outlet 1 as the low-standard outlet and to outlet 2 as the high-standard one. We start by analyzing the average participation of an outlet on the distribution of scoops and how it relates to the audiences and editorial standards of the two outlets. ${ }^{13}$ Let $\widetilde{A}_{1}=\frac{A_{1}}{A_{1}+A_{2}}$ denote the normalized audience of the low-standard outlet, where $A_{1}$ and $A_{2}$ are any arbitrary values. From (7) we have:

$$
\begin{aligned}
& F_{1, \text { news }}=\left(\mu_{2}-\mu_{1}\right)+\widetilde{A}_{1}\left(1-\mu_{2}\right), \\
& F_{2, \text { news }}=\left(1-\widetilde{A}_{1}\right)\left(1-\mu_{2}\right) .
\end{aligned}
$$

Figure 1 represents expressions $F_{1 \text {,news }}$ and $F_{2 \text {,news }}$ as a function of $\widetilde{A}_{1}$. Note that $F_{1 \text {,news }}$ is increasing in $\widetilde{A}_{1}$ and $F_{2}$,news is decreasing in $\widetilde{A}_{1}$. Additionally, note that if $1-\mu_{2}>\mu_{2}-\mu_{1}$ the two functions cross at $A_{F}=\frac{1}{2}-\frac{\mu_{2}-\mu_{1}}{2\left(1-\mu_{2}\right)}$, with $A_{F} \in\left(0, \frac{1}{2}\right)$. Otherwise, $F_{1 \text {,news }}$ is always above $F_{2 \text {,news }}$, i.e., $A_{F} \leq 0$. Because the latter requires $\mu_{2}-\mu_{1}>1-\mu_{2}$, or equivalently $\mu_{2}>\frac{1+\mu_{1}}{2}$, we observe that if $\mu_{1}$ and $\mu_{2}$ are different enough the low-standard outlet will always receive a higher share of the news. By contrast, if $\mu_{2}<\frac{1+\mu_{1}}{2}$, which requires $\mu_{2}$ and $\mu_{1}$ not to be very different, there are values of $\widetilde{A}_{1}$ for which the high-standard outlet receives a higher share of the news. This is the case when $0<\widetilde{A}_{1}<A_{F}$.

Figure 1 also shows three regions delimited by the values of $\widetilde{A}_{1}$ for which $F_{1 \text {,news }}=$ $F_{2 \text {,news }}$ on the one hand, and $A_{1}=A_{2}$ on the other hand. Note that by definition the latter occurs when $\widetilde{A}_{1}=1 / 2$. Accordingly, Region I corresponds to values $\frac{1}{2}<\widetilde{A}_{1} \leq$

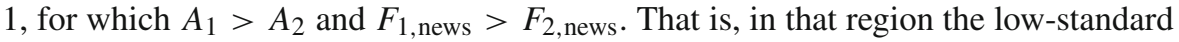
outlet leads the market in terms of both audience and news share. Region II corresponds

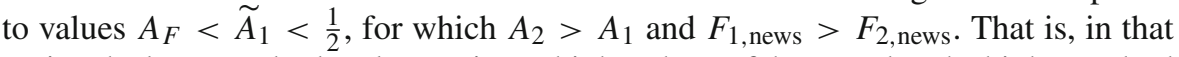
region the low-standard outlet receives a higher share of the news but the high-standard outlet has a higher audience. Finally, Region III corresponds to values $0 \leq \widetilde{A}_{1}<A_{F}$,

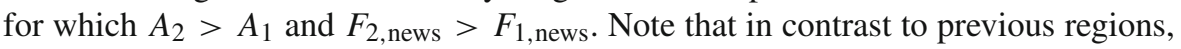
this region does not always exist, as $A_{F}$ may be smaller than zero. When it does exist, it represents a situation in which the high-standard outlet leads the market in terms of both audience and news share.

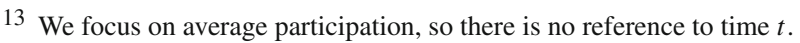


Fig. 1 We represent the share of the news of outlets 1 and 2 . The red line corresponds to the fraction of news published by the low-standard outlet (with $\left.\mu_{1}=0.2\right)$ and the blue line to the fraction of news published by the high-standard outlet (with $\left.\mu_{2}=0.4\right)$. The vertical lines at $A_{F}$ (value of $\widetilde{A}_{1}$ such that

$\left.F_{1, \text { news }}=F_{2, \text { news }}\right)$ and at $1 / 2$ (value of $\widetilde{A}_{1}$ such that $A_{1}=A_{2}$ ) delimit Regions I, II, and III described in the text

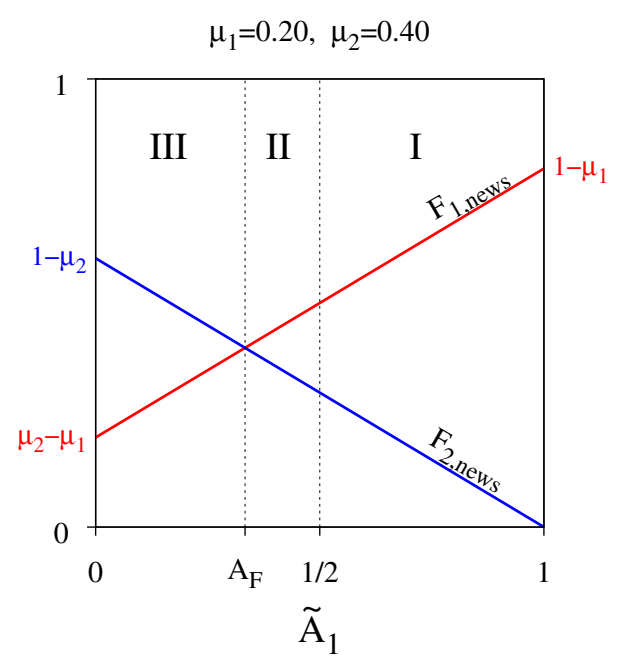

The analysis above determines the general conditions under which outlet $i$ publishes more stories than outlet $j$, with $i, j \in\{1,2\}, i \neq j$. It also determines the way in which the fraction of news published by an outlet relates to that outlet's audience. This analysis reveals that in general it is the low-standard outlet that publishes more scoops. Interestingly, this situation can occur for any pair of editorial standards, i.e., independently of whether media outlets are similar in their vetting processes for stories or not. By contrast, for the high-standard outlet to receive and publish a higher share of the news the market needs to be homogenous enough.

Next, we move into the analysis of the equilibrium of the mean-field dynamics. The stationary condition of the dynamics is $\Delta A_{i}(t)=A_{i}(t+1)-A_{i}(t)=0$ in Eq. (6). In the case of a media industry with two outlets, this defines a system of two equations. The pairs $\left(A_{1}^{*}, A_{2}^{*}\right)$ that satisfy the system are the equilibrium values. Next proposition presents the result.

\section{Proposition 1 The system has two stationary states.}

1. In the first one, $A_{2}^{*}=0$. This stationary state is unstable.

2. In the second one, $0<A_{1}^{*}<1$ and $0<A_{2}^{*}<1$. This stationary state is asymptotically stable.

Proposition 1 states that the system has two stationary states and that only the second one is stable. To see the intuition for this, note that if an outlet reaches zero audience at a time $t$, it will have no ability to attract further scoops (unless it is the low-standard outlet, in which case it receives and publishes all the scoops whose quality lies within the interval $\left.\left(\mu_{1}, \mu_{2}\right)\right)$ so it will remain with zero audience forever. Accordingly, there is a stationary state in which $A_{2}^{*}=0$. However, if we perturb the dynamics and assign a scoop to this outlet, its audience will eventually increase. Accordingly, we say that this stationary state is unstable.

Figure 2 presents the phase diagram of the system (left panel) and the vector field (right panel) for a particular case of the parameter values, in particular $\alpha=0.5$, 

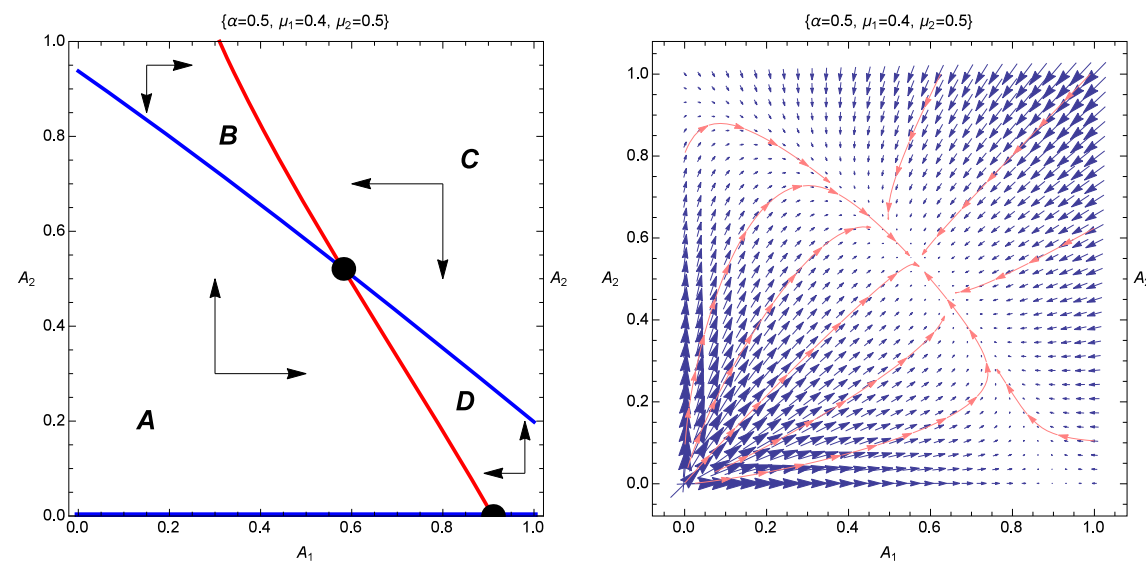

Fig. 2 The left panel represents the phase diagram of the system. The red line corresponds to the pairs $\left(A_{1}, A_{2}\right)$ such that $\Delta A_{1}(t)=0$. The blue lines to the pairs $\left(A_{1}, A_{2}\right)$ such that $\Delta A_{2}(t)=0$. When $\Delta A_{2}(t)=0$ for all $A_{1}$, in the stationary state $A_{2}^{*}=0$. When $\Delta A_{2}(t)=0$ defines a decreasing function in $A_{1}$, in the stationary state $0<A_{1}^{*}<1$ and $0<A_{2}^{*}<1$. In this case, the intersection of the described line with the red line defines four regions: A, B, C and D. The arrows in each region indicate the direction of a change in the outlets' audiences. The right panel represents the vector field. In both panels, we consider $\alpha=0.5, \mu_{1}=0.4$ and $\mu_{2}=0.5$

$\mu_{1}=0.4$ and $\mu_{2}=0.5$. The left panel shows the pairs $\left(A_{1}, A_{2}\right)$ such that $\Delta A_{1}(t)=0$ (in red) and $\Delta A_{2}(t)=0$ (in blue). As observed, there are two stationary states: In the first one $A_{2}^{*}=0$. In the second one $0<A_{1}^{*}<1$ and $0<A_{2}^{*}<1$. See Appendix $\mathrm{C}$ for the explicit expressions of $A_{1}^{*}$ and $A_{2}^{*}{ }^{14}$ The right panel presents a graphic analysis of the stability of the system. As observed, only the stationary state with $0<A_{1}^{*}<1$ and $0<A_{2}^{*}<1$ is stable.

The rest of the analysis focuses on the stable stationary state. We next seek to establish the conditions on $\alpha, \mu_{1}$ and $\mu_{2}$ for which the stable stationary values $\left(\widetilde{A}_{1}^{*}, \widetilde{A}_{2}^{*}\right)$ belong to each of the regions described, i.e., Regions I, II, and III. This is done in Propositions 2 and 3 and the discussion that follows.

First we show the existence of threshold $\hat{\alpha}_{A}$, which delimits Region I from Regions II and III. In this sense, threshold $\hat{\alpha}_{A}$ establishes which outlet receives a higher audience in equilibrium.

Proposition 2 There is a threshold $\hat{\alpha}_{A}=\frac{2}{{ }_{\sim}-\left(\mu_{1}+\mu_{2}\right)}$ such that in the stable stationary state $\widetilde{A}_{2}^{*} \geq \widetilde{A}_{1}^{*}$ if and only if $\alpha \geq \hat{\alpha}_{A}$, and $\widetilde{A}_{1}^{*}>\widetilde{A}_{2}^{*}$ otherwise.

Proposition 2 says that when societies are harsh enough with the publication of false stories, in equilibrium the outlet that obtains a higher audience will be the highstandard one. Otherwise, it will be the low-standard outlet. Note that since $\hat{\alpha}_{A}>1 / 2$, if $\alpha \leq 1 / 2$ it is always the case that $\widetilde{A}_{1}^{*}>\widetilde{A}_{2}^{*}$. That is, if the cost of publishing false stories is smaller than the reward for publishing a story, then the outlet with greater audience will always be the low-standard one, no matter whether outlets are similar in

\footnotetext{
${ }^{14}$ In Appendix $\mathrm{C}$ we give the expressions for the normalized audiences $\widetilde{A}_{1}^{*}$ and $\widetilde{A}_{2}^{*}$. The values for $A_{1}^{*}$ and $A_{2}^{*}$ are obtained by substituting $\widetilde{A}_{1}^{*}$ and $\widetilde{A}_{2}^{*}$ in Eq. (6).
} 
their vetting processes for stories or are very different. Lastly, note that $\hat{\alpha}_{A}$ is increasing in $\mu_{1}+\mu_{2}$, so if $\mu_{1}$ and $\mu_{2}$ are small enough there are values of $\alpha$ for which $\widetilde{A}_{2}^{*}>\widetilde{A}_{1}^{*}$. Note also that the higher the editorial standards of the outlets are, the harsher a society must be for the high-standard outlet to end up with a higher audience.

The next proposition shows that there is a threshold $\hat{\alpha}_{F}$ such that together with an additional condition on the outlets' editorial standards, Region III is delimited both from Regions I and II. In other words, these conditions establish which outlet receives and publishes a higher share of the news.

Proposition 3 There is a threshold $\hat{\alpha}_{F}=\frac{4-4 \mu_{2}}{5-\mu_{1}^{2}-6 \mu_{2}+2 \mu_{1} \mu_{2}}$ such that in the stable stationary state $F_{2, \text { news }}^{*} \geq F_{1 \text {,news }}^{*}$ if and only if $\alpha \geq \hat{\alpha}_{F}$ and $\mu_{2}<\frac{1+\mu_{1}}{2}$, and $F_{1, \text { news }}^{*}>F_{2 \text {,news }}^{*}$ otherwise.

Proposition 3 states that for punishment values higher than the threshold, if the editorial standards of the two outlets are not very different, in equilibrium the highstandard outlet receives and publishes a higher share of the news than the low-standard outlet. Note that $\hat{\alpha}_{F}$ is decreasing in both $\mu_{1}$ and $\mu_{2}$, thus $\hat{\alpha}_{F}>4 / 5$ always. Hence, if $\alpha \leq 4 / 5$ then $F_{1, \text { news }}^{*}>F_{2 \text {,news }}^{*}$ always, independently of the outlets' editorial standards. That is, unless society is harsh enough with the publication of false stories, the outlet receiving and publishing a higher share of the news will always be the low-standard one.

Now, we put together the results from Propositions 2 and 3 and determine the equilibrium size of Regions I, II, and III and the values of $\alpha$ for which the stable stationary values $\left(\widetilde{A}_{1}^{*}, \widetilde{A}_{2}^{*}\right)$ belong to each of the regions. To that end, observe that given a pair $\left(\mu_{1}, \mu_{2}\right)$, the equilibrium value $\widetilde{A}_{1}^{*}$ is decreasing in $\alpha$, with $\widetilde{A}_{1}^{*}(\alpha=1) \geq 0$ and $\widetilde{A}_{1}^{*}(\alpha=0) \leq 1$. Let $A_{\text {low }}=\widetilde{A}_{1}^{*}(\alpha=1)$ denote the lower limit and $A_{\text {up }}=\widetilde{A}_{1}^{*}(\alpha=0)$ denote the upper limit. ${ }^{15}$ Figure 3 below represents these ideas in two cases. In the left panel, we consider $\mu_{1}=0.2$ and $\mu_{2}=0.4$; in the right panel, we consider $\mu_{1}=0.4$ and $\mu_{2}=0.6$. The colored strips indicate the range of values of the audiences for which the equilibrium of the system belongs to Regions I, II, and III. Note that together with values $A_{F}$ and $\widetilde{A}_{1}^{*}=1 / 2$, thresholds $A_{\text {low }}$ and $A_{\text {up }}$ fully characterize Regions I, II, and III in equilibrium. ${ }^{16}$

Lastly, we conduct the exercise for the editorial standards. This is done in Fig. 4, where we determine the equilibrium size of Regions I, II, and III and the values of $\mu_{1}$ and $\mu_{2}$ for which the stable stationary values $\left(\widetilde{A}_{1}^{*}, \widetilde{A}_{2}^{*}\right)$ belong to each region. Since $\widetilde{A}_{1}^{*}>\widetilde{A}_{2}^{*} \forall \alpha \leq 1 / 2$, and $F_{1, \text { news }}^{*}>F_{2, \text { news }}^{*} \forall \alpha \leq 4 / 5$, we only consider values of $\alpha$ higher than $1 / 2$. We observe that the higher $\alpha$ is, the higher the region is in which the high-standard outlet receives a higher audience in equilibrium than the low-standard outlet. Note that this occurs in both Regions II and III. To see why, note that from Proposition 2 it is known that $\widetilde{A}_{2}^{*} \geq \widetilde{A}_{1}^{*}$ if and only if $\alpha \geq \frac{2}{4-\left(\mu_{1}+\mu_{2}\right)}$. This condition can be rewritten as $\mu_{1}+\mu_{2} \leq 4-\frac{2}{\alpha}$. Hence, the greater the punishment, the higher

\footnotetext{
15 It can be shown that at $A_{\text {low }}$, it is always the case that $F_{1 \text {,false }}=F_{2}$,false.

16 Substituting we obtain $A_{\text {low }}=\widetilde{A}_{1}^{*}(\alpha=1)=1-\frac{\left(1-\mu_{1}\right)^{2}}{2\left(1-\mu_{2}\right)^{2}}$ if $\mu_{2}<1-\frac{1-\mu_{1}}{\sqrt{2}}$, otherwise $A_{\text {low }}=0$.

We also obtain $A_{\text {up }}=\widetilde{A}_{1}^{*}(\alpha=0)=\frac{1+\mu_{1}-2 \mu_{2}+\sqrt{1-6 \mu_{1}+\mu_{1}^{2}+4\left(1+\mu_{1}\right) \mu_{2}-4 \mu_{2}^{2}}}{4-4 \mu_{2}}$, with $1 / 2<A_{\text {up }}<1$.
} 

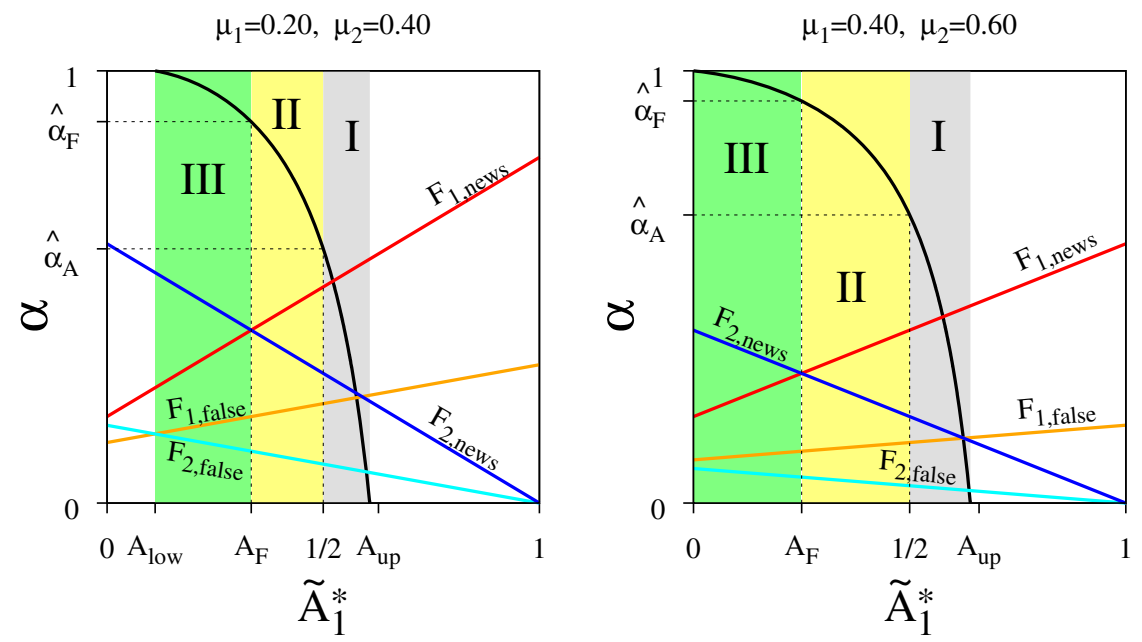

Fig. 3 We represent Regions I, II, and III in equilibrium. The left panel considers $\mu_{1}=0.2$ and $\mu_{2}=0.4$; the right panel considers $\mu_{1}=0.4$ and $\mu_{2}=0.6$. In each panel, the red line corresponds to the fraction of news published by the low-standard outlet and the blue line to the fraction of news published by the highstandard outlet. The orange and cyan lines correspond to the fraction of false stories published by the low and the high-standard outlet, respectively. The black curve gives the value of parameter $\alpha$ that is required to reach a particular equilibrium audience $\widetilde{A}_{1}^{*}$. The vertical lines at $A_{\text {low }}, A_{F}, 1 / 2$ and $A_{\text {up }}$ determine the equilibrium size of Regions I, II, and III. The left boundary $A_{\text {low }}$ corresponds to the case with no reward, i.e., $\widetilde{A}_{1}^{*}(\alpha=1)$; the right boundary $A_{\text {up }}$ to the case with no punishment, i.e., $\widetilde{A}_{1}^{*}(\alpha=0)$. Note that at $A_{\text {low }}$, the fraction of false stories published in equilibrium by the two outlets is the same

the pair of values $\left(\mu_{1}, \mu_{2}\right)$ for which $\widetilde{A}_{2}^{*} \geq \widetilde{A}_{1}^{*}$. Similarly, we observe that the higher $\alpha$ is, the higher the region is in which the high-standard outlet receives and publishes a higher share of the news. This occurs only in Region III. Lastly, we observe that Region III is never the whole region. In fact, when $\alpha=1$ there are pairs $\left(\mu_{1}, \mu_{2}\right)$ for which $\widetilde{A}_{2}^{*}>\widetilde{A}_{1}^{*}$ but $F_{1, \text { news }}^{*}>F_{2, \text { news }}^{*}$. This is the case when the two outlets have relatively different editorial standards. Accordingly, our model predicts that low-standard outlets will receive and publish a greater share of the news whenever the media industry is made up of very different media outlets.

\section{The stochastic dynamics}

In this section, we discuss some of the results obtained when we consider the stochastic dynamics described in Eq. (2). The analysis of the stochastic dynamics has two purposes: On the one hand to check the robustness of the mean-field results to the consideration of a stochastic dynamics with a number $N$ of media outlets. The idea is to study whether the relationship between audience, consumers' valuation of news and market dispersion found previously holds in the present case. Figures 7 and 8 in Appendix B show that it does for most of the insights. For the sake of conciseness, these results are relegated to the Appendix. The second purpose of the stochastic dynamics analysis is to draw up new predictions on the dynamics of the system when 

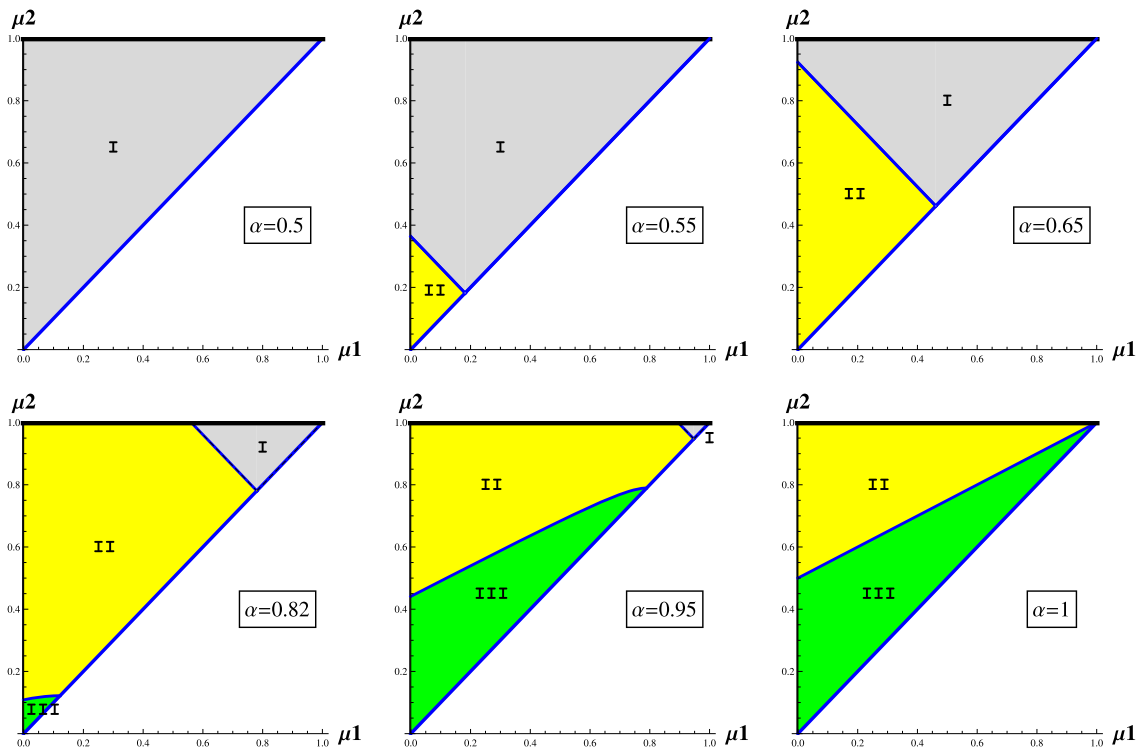

Fig. 4 We represent Regions I, II, and III in equilibrium as a function of the pairs $\left(\mu_{1}, \mu_{2}\right)$ satisfying $\mu_{1}<\mu_{2}$, for different values of parameter $\alpha$. In blue we represent the pairs $\left(\mu_{1}, \mu_{2}\right)$ for which $\widetilde{A}_{1}^{*}=\widetilde{A}_{2}^{*}$ and $F_{1, \text { news }}^{*}=F_{2, \text { news }}^{*}$ (color figure online)

there are more than two outlets. A relevant question here is what characteristics of the media industry make it more likely that a higher number of outlets will endure in the long run. Figures 5 and 6 below present results along this line. In these figures, we consider $\Phi=1 / 10$, which represents a situation in which the public needs less than a year to build a quasi-stationary opinion about a media outlet and at the same time the publication of a single scoop can have a substantial impact on an outlet's audience. ${ }^{17}$ See Appendix B for a discussion of the effect of $\Phi$ on the results.

Figure 5 represents the final audiences of a media industry with $N=5$ after a 20-year period, for a representative combination of $\mu_{1}, \ldots, \mu_{5}$ and $\alpha$. The top, center and bottom panels show high, intermediate and low punishments, respectively. Each vertical line-connecting five dots- corresponds to a single simulation, where the lowest dot corresponds to $\mu_{1}$ and the highest to $\mu_{5}$. The color of a dot represents the final audience of the outlet, as indicated in the palette. Finally, the crosses indicate outlets that loose all their audience and go out of business. The first result that can be observed is that when $\alpha$ is high there is a positive correlation between an outlet's editorial standard and its final audience. By contrast, when $\alpha$ is low the correlation is negative. The second (and new) result is that there are configurations of parameters for which the dynamics drive some outlets out of the market. ${ }^{18}$ A look at Fig. 5 suggests

\footnotetext{
17 Note that when $\Phi=1 / 10$, an outlet with an audience of $\widetilde{A}=0.9$ that publishes one false story reduces its audience by about $10 \%$ when $\alpha \sim 1$. Similarly, an outlet with an audience of $\widetilde{A}=0.1$ that publishes a true scoop almost doubles its audience when $\alpha \ll 1$.

18 Technically, this requires an outlet (say $i$ ) to reach zero audience $A_{i}=0$ at some time $t$. In this case, that outlet no longer receives scoops so from then onwards it is out of business [unless it is the outlet with
} 

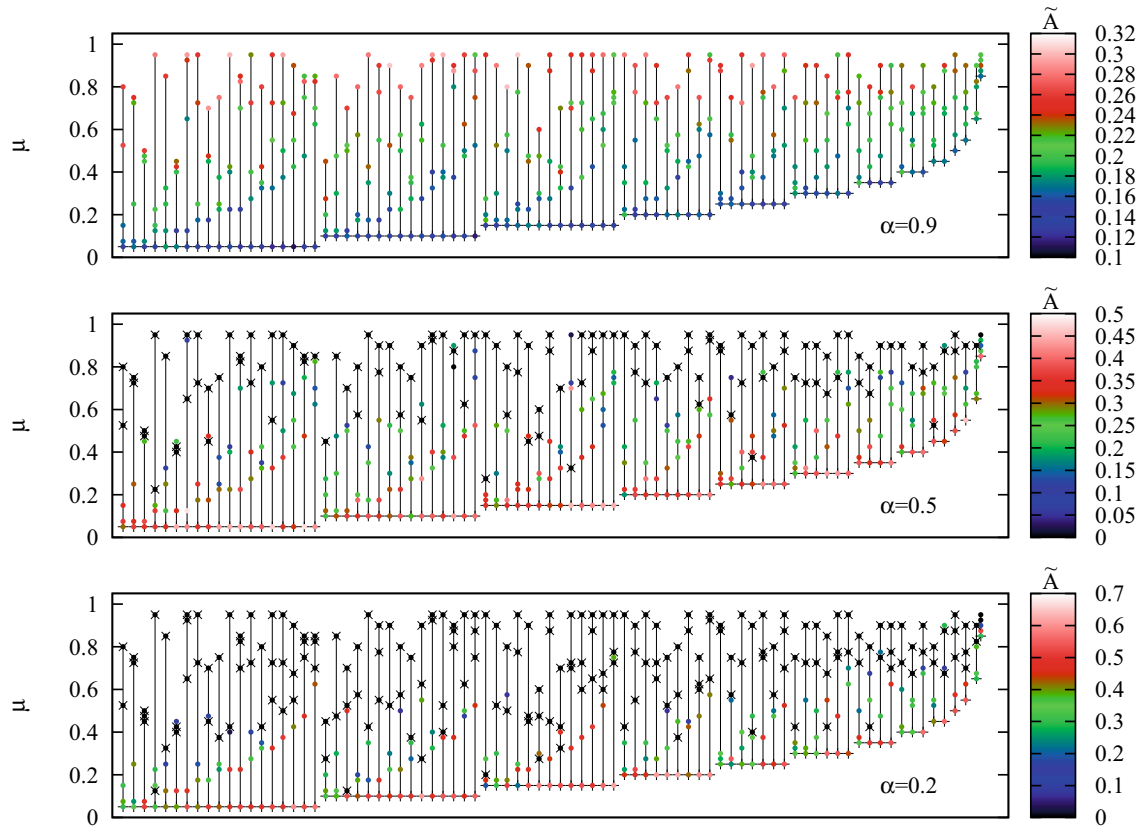

Fig. 5 We represent the audiences of $N=5$ outlets after 20 years of evolution for different combinations of the editorial standards of the outlets. The top, center and bottom panels show the audiences when $\alpha=0.9$, $\alpha=0.5$, and $\alpha=0.2$, respectively. Each vertical line-connecting five dots-corresponds to a single simulation, where the lowest dot corresponds to $\mu_{1}$ and the highest dot to $\mu_{5}$. The color of a dot represents the final audience of the outlet, as indicated in the palette. The simulations shown are a small sample of all possible $\mu_{i}$ combinations, with $i=1, \ldots, 5$. The value of $\mu_{1}$ increases from left to right to facilitate the presentation. The crosses indicate the outlets that lost all their audience and went out of business (color figure online)

that consumers' valuation of news and market dispersion may both affect the degree of competition in the long run. Figure 6 explores this question in more detail.

Figure 6 represents the probability that $m \leq N$ outlets will survive in the industry in the long run. The left and center panels represent the probability that $m \leq N$ outlets will survive as a function of $\alpha$. It considers $N=5$ (left panel) and $N=10$ (center panel). For each value of $\alpha(\alpha=0.05,0.1,0.15, \ldots 1)$, we perform twenty thousand simulations with random editorial standards and random initial audiences, and we run them for the equivalent of 20 years. Two results are worth mentioning. First, the probability of more outlets surviving in the long run seems to be positively correlated with parameter $\alpha$. Hence, the more harshly consumers punish the publication of false stories, the more outlets survive in the long run. Second, for a given $\alpha$, the number of outlets $m$ that survive presents a low dispersion and an inverted $U$-shaped form, with dispersion being higher when $\alpha \sim 0.5$ and lower when $\alpha \sim 0$ and $\alpha \sim 1$.

Footnote 18 continued

the lowest editorial standard, in which case it always publishes the scoops within the interval $\left.\left(\mu_{1}, \mu_{2}\right)\right]$. Note that in the mean-field approach, we also obtain this result, i.e., $\widetilde{A}_{2}^{*}=0$, but show that this stationary state is unstable. 

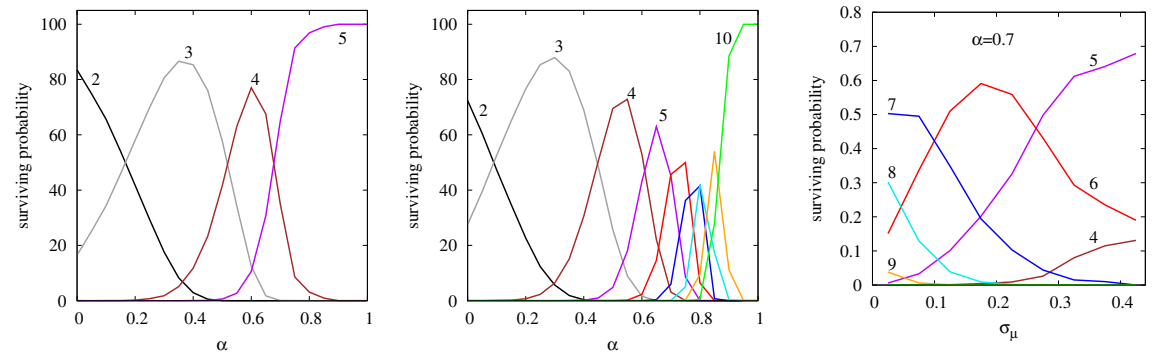

Fig. 6 The left and center panels represent the probability that $m \leq N$ media outlets will survive in the long run as a function of $\alpha$. The left panel considers $N=5$ and the center panel $N=10$. The label by each curve indicates the number of surviving outlets. The right panel represents the probability that $m \leq N$ outlets will survive in the long run as a function of the standard deviation $\sigma_{\mu}$ of the editorial standards. We consider $N=10$ and $\alpha=0.7$

The right panel represents the probability that $m \leq N$ outlets will survive in the long run as a function of the market dispersion. We define the market dispersion as the standard deviation $\sigma_{\mu}$ of the $N$ outlets' editorial standards, i.e., $\sigma_{\mu}=\sqrt{\frac{\sum_{i=1}^{N}\left(\mu_{i}-\langle\mu\rangle\right)^{2}}{N}}$. We consider $\alpha=0.7,10$ outlets and run a representative sample of all $\mu_{i}$ combinations, with $i=1, \ldots, 10$, two hundred and fifty thousand times. On average, we find that the number of outlets that survive in the industry in the long run increases as the standard deviation of the outlets' editorial standards decreases. Hence, the more homogeneous outlets are, the tougher the competition in the industry will be in the long run. ${ }^{19}$

\section{Conclusion}

This paper presents a dynamic model of competition for audience in the market for scoops. Our main contribution is to pin down two important variables to explain the dynamics: The consumers' valuation of news and the composition of the market. We obtain that the harsher consumers are with the publication of false stories, the more likely it is that high-standard media outlets receive higher equilibrium audiences. In contrast, when consumers are especially generous with outlets that break news and do not penalize them when their stories are shown to be false, our prediction is that the market will be dominated (in terms of both audience and share of the news) by low-standard outlets. Regarding the composition of the market, we obtain that only if media outlets are similar enough in their editorial standards for quality, it is possible that high-standard outlets publish a higher share of the news. Otherwise, it will be the low-standard outlets that lead the market.

\footnotetext{
19 Additional simulations show that though small values of $\sigma_{\mu}$ may occur for a variety of mean values $\langle\mu\rangle$, those with high values of $\langle\mu\rangle$ ensure competition $(m \sim M)$ and the publication of truthful information. Nevertheless, note that small values of $\sigma_{\mu}$ and high values of $\langle\mu\rangle$ imply high values of $\mu_{1}$, so the counterpart is that a substantial fraction of true stories remains unpublished. In this sense, societies that discourage the operation of outlets with very low editorial standards and that harshly punish the publication of false stories will enjoy high levels of competition and low levels of false stories in the long run. These results are available from the authors upon request.
} 
Although moving from theory to the real world is always difficult, we consider that our results help explain the great heterogeneity that characterizes the traditional press all over the world. Coming back to the examples discussed in Introduction, we propose an argument to explain why countries such as the USA and Spain show a positive correlation between newspapers' editorial standards, audiences (circulation) and share of the news, whereas countries such as the UK have a negative correlation. The argument relies on the consumers' valuation of news and builds on the idea that the average consumer of a newspaper in the UK is quite different from the average consumer of a newspaper in the US or Spain. The reason is the strength of the tabloid press in the UK and the distinctive features of its readership. ${ }^{20}$ Based on this, can the average UK newspaper reader be described as more lenient with the publication of false stories and/or a more eager consumer of scoops than the average reader in the US or Spain? It is difficult and possibly controversial to argue so, but if we agree on this point then the result follows. ${ }^{21}$ Namely, we have an argument to explain why countries with harsh consumers and homogeneous media industries (such as the US and Spain) have media outlets with high-quality standards, whereas countries such as the UK, where consumers are more lenient and the industry is more heterogeneous, show the opposite features. ${ }^{22}$

Beyond the traditional press, we believe that our model also sheds light on the use of social media as a source of information, the lack of fact-checking and editorial judgment that characterize social media platforms, and the subsequent proliferation of fake news. In line with the discussion above, our work contributes to this debate by highlighting the importance of consumers' behavior in monitoring fake news. In particular, it suggests that as long as consumers continue rewarding the mere publication of news (with shares, likes, comments and such) and not penalizing the publication of false stories, there is little to be done in this respect.

Our analysis also draws up predictions as to how many outlets will survive in the media industry in the long run. In this respect, our results show that the harsher consumers are and/or the more homogeneous outlets are, the more outlets will survive in the long run. Otherwise, the dynamics will drive outlets out of the industry, thus reducing competition over time.

To some extent, the results on the reward/punishment scheme and the number of outlets operating in the market in the long run are related to Duverger's Law, which describes a nexus between electoral systems and the number of parties in a political system. More precisely, Duverger's Law states that a plurality rule tends to favor a two-party system, whereas proportional representation tends to favor a multi-party system. In our view, the determinants of this result are similar to those of our case. Namely, high rewards lead to inequalities and the emergence of large-scale players

\footnotetext{
20 According to a study by ACORN, which classifies consumers according to their social status and shows an outline of their preferences, tabloids are mostly read by individuals in difficult circumstances, say low skilled workers and/or unemployed people. See The Consumer Classification, 2014. See also the YouGovProfile tool, an app developed by YouGov, for more information on the audience profiles of USA and UK newspapers.

21 Note that the existence of a vigorous tabloid press can be seen as supporting our argument.

22 Note that the existence of the tabloid press makes the UK newspaper industry much more heterogeneous (in terms of editorial standards) than the media industry in other countries.
} 
and so erode competition, whereas low rewards leave room for more players and foster competition for longer. In our view, this conclusion extends beyond the current setup and can help explain other relevant empirical phenomena.

This study makes a number of assumptions. First, we consider media outlets and consumers that behave according to predetermined behavioral rules. Although the model that we present in Appendix A seeks to show the rationale behind these assumptions, we consider it an interesting, though complex, exercise, to analyze the long run outcomes of a dynamic model with strategic agents. Other assumptions in the model include the absence of ideological aspects and prices. These assumptions greatly simplify the analysis, as they limit the number of variables that may affect the probability of receiving a scoop. A more general model that seeks to relax these assumptions should carefully reformulate this rule. We consider this an interesting issue for future research.

\section{Compliance with ethical standards}

Conflict of interest The authors have no conflict of interest.

Open Access This article is distributed under the terms of the Creative Commons Attribution 4.0 International License (http://creativecommons.org/licenses/by/4.0/), which permits unrestricted use, distribution, and reproduction in any medium, provided you give appropriate credit to the original author(s) and the source, provide a link to the Creative Commons license, and indicate if changes were made.

\section{A Appendix: Micro-foundations of the model}

In the main body of the paper, agents make decisions based on behavioral assumptions. The assumptions that we make with respect to the behavior of media outlets are that (i) a media outlet which does not receive a scoop never publishes a story; and (ii) a media outlet which receives a scoop publishes it only if it is of sufficiently high quality, i.e., meets the outlet's editorial standard. With respect to consumers, we assume that consumers reward media outlets that publish stories and punish those outlets that are shown to have printed false stories.

The objective of this section is to show the rationale behind these behavioral assumptions, i.e., to show that these behaviors can be optimal for strategic agents. In this sense, there are two questions of interest: The first is to study whether a strategic media outlet can find it optimal to behave as described by the behavioral rule. The second is to understand what kind of consumer preferences lead to the consumer behavior described.

The model and the results in our previous paper Andina-Díaz and García-Martínez (2018) (hereafter referred to as AG) provide an answer to these questions. It shows that the behavioral assumptions of the present paper are equilibrium strategies of the strategic agents of the one-shot game in AG. Hence, in the discussion that follows we describe the main ideas of that paper that help us rationalize the behavioral assumptions made in the present work. To that end, it suffices to focus on the monopoly case analyzed in AG, which considers a media outlet and a mass of consumers who are uncertain about the type of the outlet, i.e., whether it is a good or a normal type. The 
media outlet seeks to build a reputation for being good. The idea is that media outlets which are perceived as good by consumers receive higher audience/circulation levels; hence, the objective of maximizing audience (as in the present paper) and the objective of maximizing reputation for being good (as in AG) go hand in hand.

There is underlying uncertainty as to the state of the world, and the role of the media outlet (which is better informed on the state than consumers) is to inform consumers about the state. Consumers value information. One possible reason is that consumers have to make an investment decision and the better the information that they hold, the more accurate the investment decision (and hence the higher the expected return from the investment) will be. We consider that the good type media outlet receives a perfectly informative signal and that the normal type receives an imperfect but informative signal. Consumers do not know the type of the media outlet but have a prior on the media outlet being a good type. The media outlet receives a signal and chooses a report. There are two signals, one indicating that there is a scoop and the other indicating that there is no scoop; there are two possible reports, to publish a scoop and not to publish a scoop. In line with the assumption in the present paper, in AG we assume that when the media outlet publishes a scoop, consumers (can) learn the state of the world; however, when the media outlet does not publish a scoop consumers do not learn the state. We say that in this case there is no feedback. Consumers observe the media outlet's report and the possible feedback about the state and, based on that information, update their belief about the media outlet's type.

Having described the main ingredients of the model, we are now in position to present the results that micro-found the behavioral assumptions of the present paper. With respect to our first question, Proposition 1 in AG shows that, in the equilibrium of the game, a normal media outlet that does not receive a scoop never publishes a story and that a normal media outlet that receives a scoop publishes it (with positive probability) only if the scoop is of a certain quality, i.e., it is above the threshold that marks the outlet's editorial standard. ${ }^{23}$ Note that this is precisely what the behavioral assumption in the present paper prescribes for the behavior of media outlets. ${ }^{24}$

With respect to our second question, the model in AG considers consumers who value information. Next, we note that considering this consumer preferences suffices to engender an equilibrium behavior that supports the behavioral assumption made in the present work, which implicitly defines the market share updating rule described in Eq. (1). To see this, note that according to the description of the model in AG there are four possible situations under which a consumer updates his belief about the type of the media outlet: (i) The outlet does not publish a scoop and there is no feedback; (ii) the outlet publishes a scoop and there is no feedback; (iii) the outlet publishes a scoop, there is feedback and consumers learn that the story is true; and iv) the outlet publishes a scoop, there is feedback and consumers learn that the story is false. It can easily be proved that unless a media outlet publishes a false scoop, in the equilibrium of AG, Bayesian consumers assign a higher probability to the outlet being good after

\footnotetext{
23 In equilibrium, a good type always follows its signal. That is, if the outlet does not receive a scoop it never publishes a story, and if it receives a scoop it always publishes a story.

24 For this equilibrium to exist in AG, we only need rational consumers who update beliefs and who consider that there are media outlets of good type.
} 
the publication of a scoop than after the non-publication of a scoop. ${ }^{25}$ This means that the expected payoff of an outlet is higher in case (ii) or case (iii) than in case (i). This result micro-founds the assumption in the present work that consumers reward the publication of stories. Additionally, it can also be proved that in equilibrium the probability that Bayesian consumers assign to the outlet being good in case iv) is smaller than in case (ii) or in case (iii). ${ }^{26}$ This result micro-founds the assumption in the present work that consumers penalize the publication of false stories.

Lastly, we discuss the rationale behind the weight $\alpha$ that we use to refer to the punishment for publishing a false story. The question is how to micro-found the fact of $\alpha$ taking higher or lower values. We argue that the belief of consumers about the type of the media outlet is key to the answer. To see this, note that the analysis in AG shows that the higher the consumers' belief that the outlet is a good type, the stricter the outlet will be with the publication of a scoop, i.e., the higher the outlet's editorial standard will be. The reason is that great expectations about the type of an outlet impose a harsh punishment on the outlet in case of failure. This leads the outlet to be stricter with its vetting process for stories, which results in higher editorial standards. In this sense, a situation with a high $\alpha$ illustrates a society where consumers have high expectations on the functioning of the media industry and hence one in which errors are harshly punished. By contrast, a situation with a low $\alpha$ illustrates a society where consumers do not expect a lot from the media industry and hence one in which the cost of an error is negligible.

\section{B Appendix: Additional results on the stochastic dynamics}

In this section, we present additional results on the stochastic dynamics described in Eq. (2). The results refer to the cases with $N=2$ and $N>2$ media outlets.

Our objective here is to test the predictions of the mean-field analysis under a stochastic dynamics. Note that by construction, the asymptotic behavior of the stochastic dynamics may be expected to consists of a stochastic fluctuation around the equilibrium mean-field values $\widetilde{A}_{i}^{*}$. Hereafter we refer to this fluctuating asymptotic behavior as the quasi-stationary state. Our results show that the time that it takes to reach the quasi-stationary state and the amplitude of the fluctuations around the mean-field equilibrium crucially depend on parameter $\Phi$, which controls the impact of the publication of a single scoop on the audience of the outlets. In fact, note that $\Phi$ represents how much society rewards and punishes the publication of true and false stories via variations in audience. As expected, the amplitude of the fluctuations is proportional to $\Phi$, whereas the characteristic time taken to reach the quasi-stationary state is inversely proportional to $\Phi$.

Now we consider the results. Figure 7 shows the dynamics over a 20 -year period of an industry with $N=2$ media outlets, with editorial standards $\mu_{1}=0.3$ (in red) and $\mu_{2}=0.5$ (in blue). The top panels consider a society with $\alpha=0.95$, and the

\footnotetext{
25 The result follows from the fact that, in equilibrium, the good type media outlet is more likely to publish a scoop than the normal type.

26 This result follows from the fact that, in equilibrium, the good type media outlet never publishes a false story.
} 
bottom panels a society with $\alpha=0.2 .^{27}$ The effect of $\Phi$ on the dynamics of the audience of an outlet is observed in the top-left and bottom-left panels of the figure. The left panels represent the evolution of the outlets' audiences, $\widetilde{A}_{1}(t)$ and $\widetilde{A}_{2}(t)$. Each panel represents four curves (two for each outlet). The curves with large fluctuations correspond to the case with $\Phi=1 / 10$, whereas the smooth ones correspond to the case with $\Phi=1 / 200$. The (stable) stationary states of the mean-field model are indicated with the two starred dots at the last time step, $t=20$. As stated, the quasi-stationary states fluctuate around the stable stationary states of the mean-field approach. It can be observed that in the top panel it takes about 20 years to reach the quasi-stationary state when $\Phi=1 / 200$, whereas if $\Phi=1 / 10$ the stories published during the first few months suffice for this state to be reached. Note that the amplitude of the stochastic fluctuations is greater when $\Phi=1 / 10$ than when $\Phi=1 / 200$. In the latter case, the quasi-stationary state closely matches the stable stationary state $\left(\widetilde{A}_{1}^{*}, \widetilde{A}_{2}^{*}\right)$. In the bottom panel convergence is faster. The reason is twofold: The initial audience figures are closer to $\left(\widetilde{A}_{1}^{*}, \widetilde{A}_{2}^{*}\right)$ and the punishment parameter $\alpha$ is small. ${ }^{28}$ Finally, a comparison of the top and the bottom panels reveals that when the punishment for publishing false stories is high (top panel) the high-standard outlet obtains more audience, whereas when the punishment is low (bottom panel), it is the other way round. This is the same result that we obtained in the mean-field approach.

The center panels show the evolution of the fraction of scoops published by each

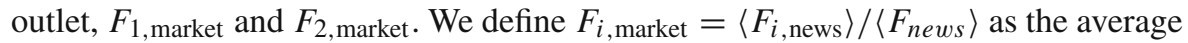
fraction of scoops published by outlet $i$ over the previous 100 days. A comparison of the top and the bottom panels shows that when $\alpha$ is low (bottom panel) the market is dominated by the low-standard outlet. By contrast, when the punishment is high (top panel) the market is dominated by the high-standard outlet. Again, this is in line with the results in the mean-field dynamics.

The right panels show the relationship between the audience of an outlet $\left\langle\widetilde{A}_{i}\right\rangle$ and the fraction of false stories published by it, defined as $p_{i, \text { false }}=\left\langle F_{i, \text { false }}\right\rangle /\left\langle F_{i \text {, news }}\right\rangle$. Again, both $\left\langle\widetilde{A}_{i}\right\rangle$ and $p_{i}$, false are calculated as average values over the previous 100 days. The results show that when the punishment is high (top panel), the probability of the outlet with the higher audience publishing false stories is low. The opposite occurs when the punishment is low (bottom panel). This is because in the latter case it is the low-standard outlet that obtains more audience. Note that the total number of false stories published in the industry depends only on $\mu_{1}$, but the way in which they are distributed between the outlets also depends on $\alpha$ and $\mu_{2}$.

The results for $N>2$ show similar patterns. They are shown in Fig. 8, which considers $N=5$. Again, the top panels consider $\alpha=0.95$ and the bottom panels $\alpha=0.2$. The editorial standards of the five media outlets are $0.15,0.30,0.45,0.60$, and 0.75 , represented in red, blue, magenta, orange, and green, respectively. As in the case with two outlets, when the punishment is high (top panels), audience is observed to be positively correlated with editorial standards and the opposite occurs when the punishment is low. The result for the fraction of scoops published by an outlet is also

\footnotetext{
27 All the panels in the figure consider the same initial conditions, which we chose far from the equilibrium values.

28 From Eq. (2), note that the average change in an outlet's audience due to the publication of a scoop decreases in $\alpha$.
} 

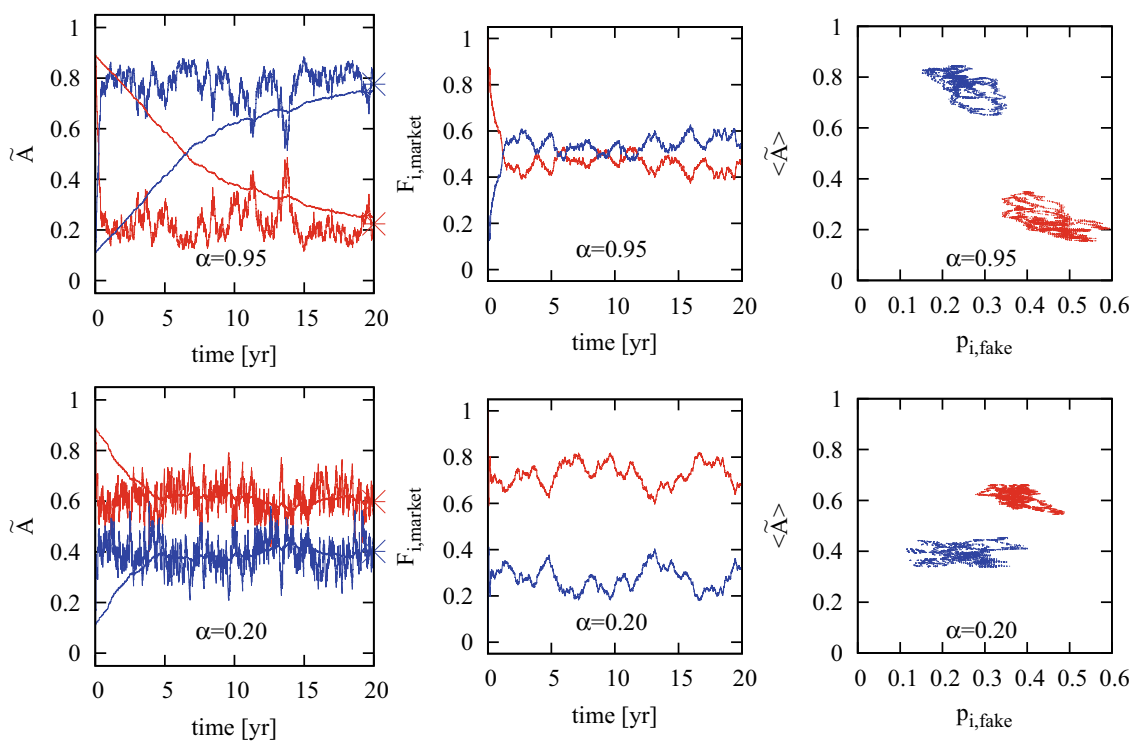

Fig. 7 We represent a media industry with $N=2$ and editorial standards $\mu_{1}=0.3$ (red) and $\mu_{2}=0.5$ (blue) over a 20-year period. The top panels correspond to a situation with a high punishment $(\alpha=0.95)$, and the bottom panels to a situation with a low punishment $(\alpha=0.2)$. The left panels show the evolution of the two outlets' audiences. The curves with large fluctuations correspond to the case $\Phi=1 / 10$, and the smooth ones to the case $\Phi=1 / 200$. The center panels show the evolution of the two fraction of news of the two outlets, averaged during the previous 100 days. The right panels show the relationship between the audience of an outlet and the fraction of false stories published by it, averaged during the previous 100 days (color figure online)

in line with those with $N=2$. Namely, the higher the punishment, the smaller the power of the outlets with low standards. However, note that when $N=5$, the outlets with low editorial standards continue to lead the market, even if the punishment is high.

\section{Appendix: Proofs}

We first present the expression for the normalized equilibrium audiences $\widetilde{A}_{1}^{*}$ and $\widetilde{A}_{2}^{*}$. From Eq. (6), writing the expression in terms of the normalized audience $\widetilde{A}_{1}=\frac{A_{1}}{A_{1}+A_{2}}$, we obtain:

$\tilde{A}_{1}^{*}=\frac{2-\alpha \mu_{1}^{2}+2 \alpha \mu_{2}^{2}-\alpha+2 \mu_{1}-4 \mu_{2}+\sqrt{\left(2-\alpha \mu_{1}^{2}+2 \alpha \mu_{2}^{2}-\alpha+2 \mu_{1}-4 \mu_{2}\right)^{2}+16(\alpha-1)\left(\mu_{2}-1\right)\left(\alpha \mu_{2}+\alpha-2\right)\left(\mu_{1}-\mu_{2}\right)}}{4\left(\mu_{2}-1\right)\left(\alpha \mu_{2}+\alpha-2\right)}$, $\widetilde{A}_{2}^{*}=1-\widetilde{A}_{1}^{*}$.

The rest of the Appendix contains the proofs. 

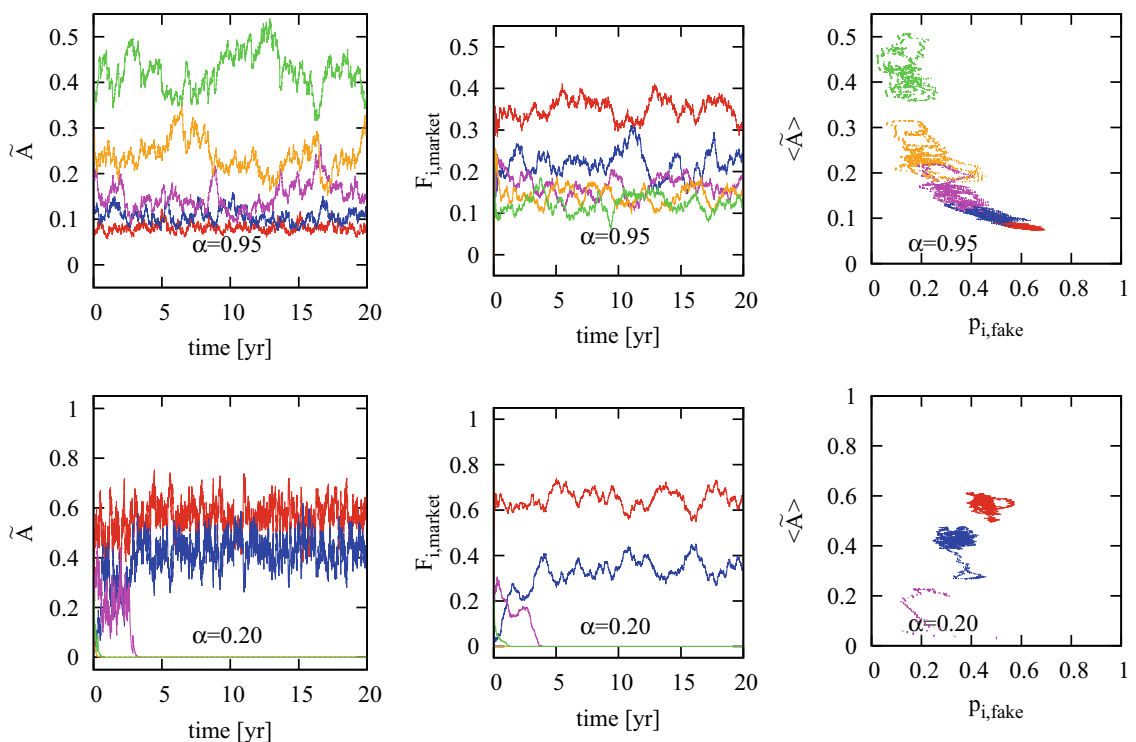

Fig. 8 Same as Fig. 7 but for five media outlets with editorial standards $\mu_{1}=0.15, \mu_{2}=0.3, \mu_{3}=0.45$, $\mu_{4}=0.6$ and $\mu_{5}=0.75$, represented in red, blue, magenta, orange, and green, respectively. The top panels consider $\alpha=0.95$ and the bottom panels $\alpha=0.2$ (color figure online)

Proof of Proposition 1 Equation (6) can be rewritten as:

$$
\begin{aligned}
& A_{1}(t+1)=f_{1}\left(A_{1}(t), A_{2}(t)\right), \\
& A_{2}(t+1)=f_{2}\left(A_{1}(t), A_{2}(t)\right),
\end{aligned}
$$

where $f_{i}:[0,1] \times[0,1] \backslash\{(0,0)\} \longrightarrow[0,1]$ for $i=1,2$.

After some algebra, we can obtain the explicit functions from expression (6). Using (7), (8) and (9), they are:

$$
\begin{aligned}
f_{1}\left(A_{1}, A_{2}\right)= & \frac{\alpha\left(1-\mu_{2}^{2}\right) A_{1}^{2}}{2\left(A_{1}+A_{2}\right)^{2}}+\frac{\left(1-\mu_{2}\right) A_{1}\left(1-\alpha-\frac{A(1)}{A_{1}+A_{2}}\right)}{A_{1}+A_{2}} \\
& +\frac{\alpha\left(\mu_{2}^{2}-\mu_{1}^{2}\right) A_{1}}{2\left(A_{1}+A_{2}\right)}+\left(\mu_{2}-\mu_{1}\right)\left(1-\alpha-\frac{A_{1}}{A_{1}+A_{2}}\right)+\frac{A_{1}}{A_{1}+A_{2}}, \\
f_{2}\left(A_{1}, A_{2}\right)= & \frac{\alpha\left(1-\mu_{2}^{2}\right) A_{2}^{2}}{2\left(A_{1}+A_{2}\right)^{2}}+\frac{\left(1-\mu_{2}\right) A_{2}\left(-\alpha-\frac{A_{2}}{A_{1}+A_{2}}+1\right)}{A_{1}+A_{2}}+\frac{A_{2}}{A_{1}+A_{2}} .
\end{aligned}
$$

Let us define

$$
\begin{aligned}
& \bar{F}_{1}\left(A_{1}, A_{2}\right)=f_{1}\left(A_{1}, A_{2}\right)-A_{1}, \\
& \bar{F}_{2}\left(A_{1}, A_{2}\right)=f_{2}\left(A_{1}, A_{2}\right)-A_{2} .
\end{aligned}
$$


Note that the sign of $\bar{F}_{i}$ determines whether $A_{i}$ increases, decreases or remains constant in the next period. After some algebra we get:

$$
\begin{aligned}
& \bar{F}_{1}\left(A_{1}, A_{2}\right) \\
& =-\frac{2 A_{1}^{3}+A_{1}\left(\alpha\left(\mu_{1}-1\right)^{2}+4 A_{2}-2\right)+A_{1} A_{2}\left(\alpha\left(\left(\mu_{1}-4\right) \mu_{1}-\left(\mu_{2}-2\right) \mu_{2}+2\right)+2\left(\mu_{1}+A_{2}-2\right)\right)-2(\alpha-1) A_{2}\left(\mu_{1}-\mu_{2}\right)}{2\left(A_{1}+A_{2}\right)^{2}}, \\
& \bar{F}_{2}\left(A_{1}, A_{2}\right)=-A_{2} \frac{\left(2 A_{1}^{2}+2 A_{1}\left(-\alpha \mu_{2}+\alpha+\mu_{2}+2 A_{2}-2\right)+A_{2}\left(\alpha\left(\mu_{2}-1\right)^{2}+2\left(A_{2}-1\right)\right)\right)}{2\left(A_{1}+A_{2}\right)^{2}} .
\end{aligned}
$$

From $\bar{F}_{2}$, it follows that if $A_{2}=0$, then $\bar{F}_{2}\left(A_{1}, A_{2}\right)=0$.

Remark 1 Let's define the function $A_{2}=g_{3}\left(A_{1}\right)$ as $g_{3}\left(A_{1}\right)=0$. Thus $\bar{F}_{2}\left(A_{1}, g_{3}\left(A_{1}\right)\right)$ $=0$.

Note that the sing of $\bar{F}_{1}$ and $\bar{F}_{2}$ are given by the sign of their numerators. Let:

$$
\begin{aligned}
F_{1}\left(A_{1}, A_{2}\right)= & -2 A_{1}^{3}-A_{1}^{2}\left(\alpha\left(1-\mu_{1}\right)^{2}\right. \\
& \left.+4 A_{2}-2\right)-A_{1} A_{2}\left(\alpha \left(\left(\mu_{1}-4\right) \mu_{1}\right.\right. \\
& \left.\left.-\left(\mu_{2}-2\right) \mu_{2}+2\right)+2\left(\mu_{1}+A_{2}-2\right)\right) \\
& +2(\alpha-1) A_{2}^{2}\left(\mu_{1}-\mu_{2}\right), \\
F_{2}\left(A_{1}, A_{2}\right)= & -\left(2 A_{1}^{2}+2 A_{1}\left(-\alpha \mu_{2}+\alpha+\mu_{2}+2 A_{2}-2\right)\right. \\
& \left.+A_{2}\left(\alpha\left(\mu_{2}-1\right)^{2}+2\left(A_{2}-1\right)\right)\right) .
\end{aligned}
$$

Next, Lemma 1 shows that there is a function $g_{1}\left(A_{1}\right)$ such that $F_{1}\left(A_{1}, g_{1}\left(A_{1}\right)\right)=0$ and Lemma 2 shows that there is a function $g_{2}\left(A_{1}\right)$ such that $F_{2}\left(A_{1}, g_{2}\left(A_{1}\right)\right)=0$.

Lemma 1 The equation $F_{1}\left(A_{1}, A_{2}\right)=0$ defines a strictly decreasing function $A_{2}=$ $g_{1}\left(A_{1}\right)$ such that for all $A_{2} \in(0,1), g_{1}^{-1}\left(A_{2}\right) \in(0,1)$.

Proof The roots of equation $F_{1}\left(A_{1}, A_{2}\right)=0$ are too complex to work with. Hence, we take a different approach.

First, we solve the equation $F_{1}\left(A_{1}^{0}, A_{2}=0\right)=0$.

There is only one solution and it is: $A_{1}^{0}=1-\frac{1}{2} \alpha\left(1+\mu_{1}^{2}-2 \mu_{1}\right)$, with $0<A_{1}^{0}<1$. Therefore, if there is a function $A_{2}=g_{1}\left(A_{1}\right)$, then $g_{1}\left(A_{1}^{0}\right)=0$. In addition, $F_{1}\left(A_{1}=\right.$ $\left.1, A_{2}=0\right)=-2+\left(\alpha\left(1-\mu_{1}\right)^{2}-2\right)<0$. The following remark summarizes this result.

Remark 2 Let $A_{1}^{0}=1-\frac{1}{2} \alpha\left(1+\mu_{1}^{2}-2 \mu_{1}\right) \in(0,1)$. Then $F_{1}\left(A_{1} \geq A_{1}^{0}, A_{2}=0\right) \leq$ 0 .

Second, we show that equation $F_{1}\left(A_{1}^{1}, A_{2}=1\right)=0$ has only one solution.

To see this, note that $F_{1}\left(A_{1}, A_{2}=1\right)$ is concave in $A_{1}$, as $\frac{\partial^{2} F_{1}\left(A_{1}^{1}, A_{2}=1\right)}{\partial A_{1}^{2}}=$ $-2 \alpha\left(1-\mu_{1}\right)^{2}-12 A_{1}-4<0$. In addition,

$$
\begin{aligned}
F_{1}\left(A_{1}=0, A_{2}=1\right)= & 2(1-\alpha)\left(\mu_{2}-\mu_{1}\right)>0 \text { and, } \\
F_{1}\left(A_{1}=1, A_{2}=1\right)= & -2\left(1-\mu_{2}\right)-\alpha\left(3-\mu_{2}^{2}\right) \\
& -4\left(\alpha \mu_{2}-(2 \alpha-1) \mu_{1}\right)-2 \alpha \mu_{1}^{2}<0 .
\end{aligned}
$$


Therefore, there is a unique $A_{1}^{1}$ such that $F_{1}\left(A_{1}^{1}, A_{2}=1\right)=0$. Consequently, if there is a function $A_{2}=g_{1}\left(A_{1}\right)$, then $g_{1}\left(A_{1}^{1}\right)=1$. The following remark summarizes this result.

Remark 3 There is $A_{1}^{1} \in(0,1)$, such that $F_{1}\left(A_{1} \geq A_{1}^{1}, A_{2}=1\right) \leq 0$.

Let $\hat{A}_{1}=\left(\mu_{2}-\mu_{1}\right)(1-\alpha)$. The following result relates thresholds $A_{1}^{0}, A_{1}^{1}$ and $\hat{A}_{1}$, showing that if $A_{1}<\hat{A}_{1}$ then $F_{1}\left(A_{1}, A_{2}\right)$ is convex in $A_{2}$ (it is concave if $A_{1}>\hat{A}_{1}$.

Claim 1 It holds that $0<\hat{A}_{1}<A_{1}^{1}<A_{1}^{0}<1$.

Proof First, note that $\frac{\partial^{2} F_{1}\left(A_{1}, A_{2}\right)}{\partial A_{2}^{2}}=-4 A_{1}+4\left(\mu_{2}-\mu_{1}\right)(1-\alpha) \geq 0$ if $A_{1} \leq$ $\left(\mu_{2}-\mu_{1}\right)(1-\alpha)$. Let $\hat{A}_{1}=\left(\mu_{2}-\mu_{1}\right)(1-\alpha)$.

Second, note that since

$$
\begin{aligned}
F_{1}\left(A_{1}=A_{1}^{0}, A_{2}=1\right)= & -\frac{1}{2} \alpha^{2}\left(\mu_{1}-1\right)^{2}\left(\mu_{1}^{2}+\left(\mu_{2}-2\right) \mu_{2}\right) \\
& +\alpha\left(\mu_{1}^{3}+\mu_{1}+\mu_{2}^{2}-4 \mu_{2}+1\right)-4 \mu_{1}+2 \mu_{2}-2<0,
\end{aligned}
$$

from Remark 3 it follows that $A_{1}^{1}<A_{1}^{0}$.

Third, note that since $F_{1}\left(A_{1}=\hat{A}_{1}, A_{2}=1\right)=(1-\alpha)\left(\mu_{2}-\mu_{1}\right)\left(4-2(1-\alpha)^{2}\left(\mu_{2}-\right.\right.$ $\left.\left.\mu_{1}\right)^{2}-(1-\alpha)\left(\mu_{2}-\mu_{1}\right)\left(\alpha\left(1-\mu_{1}\right)^{2}+2\right)-\alpha\left(\left(\mu_{1}-4\right) \mu_{1}-\left(\mu_{2}-2\right) \mu_{2}+2\right)-2 \mu_{1}\right)>0$, from Remark 3 it follows that $\hat{A}_{1}<A_{1}^{1}$.

Therefore, it is possible to divide the set $A_{1} \times A_{2}=[0,1] \times[0,1] \backslash\{(0,0)\}$ into four areas and study the function $F_{1}\left(A_{1}, A_{2}\right)$ in each of them. This is done next:

1. Area $\mathbf{1}=\left\{\left(A_{1}, A_{2}\right)\right.$ such that $\left.A_{1}<\hat{A}_{1}, A_{2} \in(0,1)\right\}$.

Note that $F_{1}\left(A_{1}, A_{2}\right)$ is continuous and convex in $A_{2}$. Additionally, since $\hat{A}_{1}<A_{1}^{1}$, $F_{1}\left(A_{1}<\hat{A}_{1}, A_{2}=1\right)>0$ and $F_{1}\left(A_{1}<\hat{A}_{1}, A_{2}=0\right)>0$. See Remark 3 and Claim 1. Consequently, if $\left.\frac{\partial F_{1}\left(A_{1}, A_{2}\right)}{\partial A_{2}}\right|_{A_{2}=0}<0$, then $F_{1}\left(A_{1}, A_{2}\right)$ is always greater than zero in this area. It is straightforward to show that

$$
\left.\frac{\partial F_{1}\left(A_{1}, A_{2}\right)}{\partial A_{2}}\right|_{A_{2}=0}=\left(4 \alpha \mu_{1}-2 \mu_{1}-2 \alpha-2 \alpha \mu_{2}-\alpha \mu_{1}^{2}+\alpha \mu_{2}^{2}+4-4 A_{1}\right) A_{1}>
$$

0 if $A_{1}<\hat{A}_{1}=\left(\mu_{2}-\mu_{1}\right)(1-\alpha)$. Consequently, $F_{1}\left(A_{1}, A_{2}\right)$ is always greater than zero in this area.

2. Area $2=\left\{\left(A_{1}, A_{2}\right)\right.$ such that $\left.\hat{A}_{1}<A_{1}<A_{1}^{1}, A_{2} \in(0,1)\right\}$.

Note that $F_{1}\left(A_{1}, A_{2}\right)$ is continuous and concave in $A_{2}$. In addition $F_{1}\left(A_{1}<\right.$ $\left.A_{1}^{1}, A_{2}=1\right)>0$ and $F_{1}\left(A_{1}<A_{1}^{1}, A_{2}=0\right)>0$. See Remark 3. Consequently $F_{1}\left(A_{1}, A_{2}\right)$ is always greater than zero in this area.

3. Area $3=\left\{\left(A_{1}, A_{2}\right)\right.$ such that $\left.A_{1}^{0}<A_{1}<1, A_{2} \in(0,1)\right\}$. 
In this area, $F_{1}\left(A_{1}, A_{2}\right)$ is continuous and concave in $A_{2}$. In addition $F_{1}\left(A_{1}>\right.$ $\left.A_{1}^{0}, A_{2}=1\right)<0$ and $F_{1}\left(A_{1}>A_{1}^{0}, A_{2}=0\right)<0$. See Remark 2. Consequently, if $\left.\frac{\partial F_{1}\left(A_{1}, A_{2}\right)}{\partial A_{2}}\right|_{A_{2}=0}<0$ for any $A_{1} \in\left(A_{1}^{0}, 1\right)$, then necessarily $F_{1}\left(A_{1}, A_{2}\right)<0$. Note that $\left.\frac{\partial F_{1}\left(A_{1}, A_{2}\right)}{\partial A_{2}}\right|_{A_{2}=0}=A_{1}\left(4 \alpha \mu_{1}-2 \mu_{1}-2 \alpha-2 \alpha \mu_{2}-\alpha \mu_{1}^{2}+\alpha \mu_{2}^{2}+4-4 A_{1}\right)=$ $A_{1}\left(4\left(\left(1-\frac{1}{2} \alpha\left(1-2 \mu_{1}+\frac{1}{2} \mu_{1}^{2}+\mu_{2}-\frac{1}{2} \mu_{2}^{2}\right)\right)-A_{1}\right)-2 \mu_{1}\right)>0$ if $A_{1}>A_{1}^{0}=$ $1-\frac{1}{2} \alpha\left(1+\mu_{1}^{2}-2 \mu_{1}\right)$.

An analysis of Areas 1,2, and 3 reveals that if the function $A_{2}=g_{1}\left(A_{1}\right)$ such that $F_{1}\left(A_{1}, A_{2}\right)=0$ exists, then it has to be defined in $A_{1}^{1}<A_{1}<A_{1}^{0}$. Next, we study Area 4.

\section{Area $4=\left\{\left(A_{1}, A_{2}\right)\right.$ such that $\left.A_{1}^{1}<A_{1}<A_{1}^{0}, A_{2} \in(0,1)\right\}$.}

First, we show that this function exists in the interval $A_{1} \in\left(A_{1}^{0}, A_{1}^{1}\right)$, and second that it is decreasing and continuous.

Note that we have already shown that $F_{1}\left(A_{1}, A_{2}\right)$ is continuous and concave in $A_{2}$ in area 4. In addition, in this area, $F_{1}\left(A_{1}, A_{2}=1\right)<0$ and $F_{1}\left(A_{1}, A_{2}=0\right)>0$. See Remarks 2, 3 and Claim 1. Consequently, for any $A_{1} \in\left(A_{1}^{0}, A_{1}^{1}\right)$, it exists a single $\bar{A}_{2}$ such that $F_{1}\left(A_{1}, A_{2}=\bar{A}_{2}\right)=0, F_{1}\left(A_{1}, A_{2}<\bar{A}_{2}\right)>0$ and $F_{1}\left(A_{1}, A_{2}>\bar{A}_{2}\right)<0$. Therefore, the function $A_{2}=g_{1}\left(A_{1}\right)$ such that $F_{1}\left(A_{1}, A_{2}\right)=0$ exists.

To prove that this function is continuous and decreasing, it suffices to prove that for any $A_{2} \in(0,1)$, there is always a unique $\bar{A}_{1}$ such that $F_{1}\left(A_{1}<\bar{A}_{1}, A_{2}\right)>0$, $F_{1}\left(A_{1}=\bar{A}_{1}, A_{2}\right)=0$ and $F_{1}\left(A_{1}>\bar{A}_{1}, A_{2}\right)<0$. First, note that $F_{1}\left(A_{1}, A_{2}\right)$ is a third degree polynomial, continuous and differentiable in $A_{1}$, with

$$
\begin{aligned}
F_{1}\left(A_{1}=0, A_{2}\right)= & 2(1-\alpha) A_{2}^{2}\left(\mu_{2}-\mu_{1}\right)>0 \\
F_{1}\left(A_{1}=1, A_{2}\right)= & -\alpha\left(1-\mu_{1}\right)^{2}-2 A_{2}^{2}\left(1+(1-\alpha)\left(\mu_{1}-\mu_{2}\right)\right) \\
& -A_{2}\left(\alpha\left(2+2 \mu_{2}-4 \mu_{1}-\mu_{2}^{2}+\mu_{1}^{2}\right)+2 \mu_{1}\right)<0 .
\end{aligned}
$$

Therefore, it exists at least one real root, so the function is continuous. Next we show that there is only one root. Hence, the function must be decreasing.

Note that since $F_{1}\left(A_{1}=0, A_{2}\right)>0, F_{1}\left(A_{1}=1, A_{2}\right)<0$ and $F_{1}$ is a continuous third degree polynomial, there could be either a single root or three roots in the interval $A_{1} \in(0,1)$ for any given $A_{2}$. If there were three roots, then $\frac{\partial F_{1}\left(A_{1}, A_{2}\right)}{\partial A_{1}}=0$ twice.

Since $\frac{\partial F_{1}\left(A_{1}, A_{2}\right)}{\partial A_{1}}=-6 A_{1}^{2}-2 A_{1}\left(\alpha\left(\mu_{1}-1\right)^{2}+4 A_{2}-2\right)-A_{2}\left(\alpha\left(\left(\mu_{1}-4\right) \mu_{1}-\right.\right.$ $\left.\left.\left(\mu_{2}-2\right) \mu_{2}+2\right)+2\left(\mu_{1}+A_{2}-2\right)\right)=0$, with roots $\frac{-2\left(\alpha\left(1-\mu_{1}\right)^{2}+4 A_{2}-2\right) \pm \sqrt{4\left(\left(\alpha\left(1-\mu_{1}\right)^{2}-2\right)^{2}+4 A_{2}^{2}+2 A_{2}\left(\alpha\left(\mu_{1}\left(\mu_{1}+4\right)-3 \mu_{2}\left(2-\mu_{2}\right)-2\right)-6 \mu_{1}+4\right)\right)}}{12}$, it $\frac{\text { can }}{\frac{-2\left(\alpha\left(1-\mu_{1}\right)^{2}+4 A_{2}-2\right)+\sqrt{4\left(\left(\alpha\left(1-\mu_{1}\right)^{2}-2\right)^{2}+4 A_{2}^{2}+2 A_{2}\left(\alpha\left(\mu_{1}\left(\mu_{1}+4\right)-3 \mu_{2}\left(2-\mu_{2}\right)-2\right)-6 \mu_{1}+4\right)\right)}}{12}}$ that $<0$.

Consequently, the derivative is zero only once. Thus, there can only be one root.

Lemma 2 The equation $F_{2}\left(A_{1}, A_{2}\right)=0$ defines a strictly decreasing function $A_{2}=$ $g_{2}\left(A_{1}\right)$ such that for all $A_{1} \in(0,1), g_{2}\left(A_{1}\right) \in(0,1)$. 
Proof

$$
\begin{aligned}
F_{2}\left(A_{1}, A_{2}\right)= & -\left(2 A_{1}+2 A_{1}\left(-\alpha \mu_{2}+\alpha+\mu_{2}+2 A_{2}-2\right)\right. \\
& \left.+A_{2}\left(\alpha\left(\mu_{2}-1\right)^{2}+2\left(A_{2}-1\right)\right)\right)=0 .
\end{aligned}
$$

The equation is a second degree polynomial in $A_{1}$ with two roots:

$$
\begin{aligned}
& -\frac{1}{4}\left(\alpha\left(1-\mu_{2}\right)^{2}-2+4 A_{1}\right) \\
& \quad \pm \frac{1}{4} \sqrt{\left(\alpha\left(1-\mu_{2}\right)^{2}-2\right)^{2}+8\left(\mu_{2}-1\right) A_{1}\left(\alpha \mu_{2}+\alpha-2\right)} .
\end{aligned}
$$

One of the roots is always negative, so the other root has to be the function $g_{2}\left(A_{1}\right)$ :

$$
\begin{aligned}
g_{2}\left(A_{1}\right)= & -\frac{1}{4}\left(\alpha\left(1-\mu_{2}\right)^{2}-2+4 A_{1}\right) \\
& +\frac{1}{4} \sqrt{\left(\alpha\left(1-\mu_{2}\right)^{2}-2\right)^{2}+8\left(\mu_{2}-1\right) A_{1}\left(\alpha \mu_{2}+\alpha-2\right)} .
\end{aligned}
$$

It satisfies $g_{2}\left(A_{1}=0\right)=-\frac{1}{2}\left(\alpha\left(1-\mu_{2}\right)^{2}-2\right)$, with $\frac{1}{2}<g_{2}\left(A_{1}=0\right)<1$, $\begin{array}{llll}g_{2}\left(A_{1}\right. & = & 1\end{array} \quad=\quad-\frac{1}{4}\left(\alpha\left(1-\mu_{2}\right)^{2}+2\right)$ $+\frac{1}{4} \sqrt{\left(\alpha\left(1-\mu_{2}\right)^{2}-2\right)^{2}+8\left(\mu_{2}-1\right)\left(\alpha \mu_{2}+\alpha-2\right)}$, with $0<g_{2}\left(A_{1}=1\right)<1$, and $\frac{\partial g_{2}}{\partial A_{1}}=\frac{\left(\mu_{2}-1\right)\left(\alpha \mu_{2}+\alpha-2\right)}{\sqrt{\left(\alpha\left(\mu_{2}-1\right)^{2}-2\right)^{2}+8\left(\mu_{2}-1\right) A_{1}\left(\alpha \mu_{2}+\alpha-2\right)}}-1<0$.

Summarizing, the function $g_{3}\left(A_{1}\right)$ is a horizontal line at $A_{2}=0$, and both $g_{1}\left(A_{1}\right)$ and $g_{2}\left(A_{1}\right)$ are strictly decreasing continuous functions satisfying the requirement that for all $A_{2} \in(0,1), g_{1}^{-1}\left(A_{2}\right) \in(0,1)$ and for all $A_{2} \in(0,1), g_{2}(A 1) \in(0,1)$. It therefore follows that $g_{1}\left(A_{1}\right)$ and $g_{2}\left(A_{1}\right)$ always cross once, defining the "inner" stationary state. Moreover, $g_{1}\left(A_{1}\right)$ and $g_{3}\left(A_{1}\right)$ also cross once at the horizontal line $A_{2}=0$, defining the "edge" stationary state. See the left panel of Fig. 2.

These functions divide the space $A_{1} \times A_{2}=[0,1] \times[0,1] \backslash\{(0,0)\}$ into four regions. It is straightforward to determine the sign of functions $F_{1}$ and $F_{2}$ in those regions and to obtain the phase diagram. See the left panel of Fig. 2. It follows that the "edge" stationary state in which $A_{2}=0$ is unstable. However, any point on the line $A_{2}=0$ converges to this edge stationary state. The following lemma states the stability of the "inner" stationary state.

Lemma 3 The inner stationary state is globally stable in $A_{1} \times A_{2}=[0,1] \times(0,1] .^{29}$

Proof Two preliminary results are needed:

Claim 2 If $A_{2}(t)<(>) g_{2}\left(A_{1}(t)\right)$, then $A_{2}(t+1)<(>) g_{2}\left(A_{1}(t)\right)$.

\footnotetext{
29 It is globally stable with the exception of the line on which $A_{2}=0$. On this line, the dynamics converge to the edge stationary state.
} 
Proof We prove the claim with " $<$ " (the proof with " $>$ " is analogous). Note that for any point below the function $g_{2}\left(A_{1}\right)$, in the next period $A_{2}$ increases since $\bar{F}_{2}\left(A_{1}, A_{2}\right)>0$ below $g_{2}\left(A_{1}\right)$. Claim 2 implies that in one period the increase in the vertical direction never crosses the function $g_{2}\left(A_{1}\right) .{ }^{30}$ This claim is equivalent to proving that for any $\bar{A}_{1} \in(0,1)$ and $A_{2}<g_{2}\left(\bar{A}_{1}\right), f_{2}\left(\bar{A}_{1}, A_{2}\right)<g_{2}\left(\bar{A}_{1}\right)$. We prove this.

First, note that $f_{2}\left(A_{1}, A_{2}=0\right)=0$, and

$\frac{\partial f_{2}\left(A_{1}, A_{2}\right)}{\partial A_{2}}=\frac{A_{1}}{\left(A_{1}+A_{2}\right)^{3}}\left((2-\alpha) A_{1}+\left(A_{2}-A_{1}\right) \mu_{2}+\alpha \mu_{2}\left(A_{1}+A_{2}\left(1-\mu_{2}\right)\right)\right)$ $>0$. Because of Lemma 2, for any $\bar{A}_{1} \in(0,1)$ there is a unique $\bar{A}_{2}$ such that $\bar{A}_{2}=g_{1}\left(\bar{A}_{1}\right)$; consequently, $f_{2}\left(\bar{A}_{1}, \bar{A}_{2}\right)=\bar{A}_{2}$. As $f_{2}$ is increasing in $A_{2}$ and $f_{2}\left(A_{1}, A_{2}=0\right)=0$, then for all $A_{2}=\left(0, \bar{A}_{2}\right), f_{2}\left(\bar{A}_{1}, A_{2}\right)<\bar{A}_{2}$.

Claim 3 If $A_{1}(t)<(>) g_{1}^{-1}\left(A_{2}(t)\right)$, then $A_{1}(t+1)<(>) g_{1}^{-1}\left(A_{2}(t)\right)$.

Proof We prove the claim with " $<$ " (the proof with " $>$ " is analogous). Note that for any point to the left of function $g_{1}\left(A_{1}\right)$, in the next period $A_{1}$ increases since $\bar{F}_{1}\left(A_{1}, A_{2}\right)>0$. Claim 2 implies that the increase in the horizontal direction never crosses function $g_{1}\left(A_{1}\right)$. This claim is equivalent to proving that for any $\bar{A}_{2} \in(0,1)$ and for any $A_{1}<g_{1}^{-1}\left(\bar{A}_{2}\right), f_{1}\left(A_{1}, \bar{A}_{2}\right)<g_{1}^{-1}\left(\bar{A}_{2}\right)$.

First note that $f_{1}\left(A_{1}=0, A_{2}\right)>0$ and $\frac{\partial f_{1}\left(A_{1}, A_{2}\right)}{\partial A_{1}}=A_{2}$ $\frac{\left(\left(2 \mu_{1}-\alpha \mu_{1}^{2}+2 \alpha \mu_{2}-\alpha \mu_{2}^{2}\right) A_{1}+\left(\alpha\left(\mu_{2}^{2}-\mu_{1}^{2}\right)+2(2-\alpha)\left(1-\mu_{2}\right)+2 \mu_{1}\right) A_{2}\right)}{2\left(A_{1}+A_{2}\right)^{3}}>0$.

Because of Lemma 1 , for any $\bar{A}_{2} \in(0,1)$ there is a unique $\bar{A}_{1}$ such that $\bar{A}_{2}=g_{1}\left(\bar{A}_{1}\right)$; consequently $f_{1}\left(\bar{A}_{1}, \bar{A}_{2}\right)=\bar{A}_{1}$. As $f_{1}$ is increasing in $A_{1}$ and $f_{2}\left(A_{1}, A_{2}=0\right)>0$, then for all $A_{1}=\left(0, \bar{A}_{1}\right), f_{1}\left(A_{1}, \bar{A}_{2}\right)<\bar{A}_{1}$.

As mentioned above, there are always four regions in $A_{1} \times A_{2}=[0,1] \times(0,1]$. See the left panel of Fig. 2. Now consider any initial point $A\left(t^{\prime}\right)=\left(A_{1}\left(t^{\prime}\right), A_{2}\left(t^{\prime}\right)\right)$ belonging to Region A. We show that the system always converges to the inner stationary state. Note that as this region is to the left of $g_{1}\left(A_{1}\right)$ and below $g_{2}\left(A_{1}\right)$, $A_{1}\left(t^{\prime}\right)<A_{1}\left(t^{\prime}+1\right)$ and $A_{2}^{\prime}(t)<A_{2}\left(t^{\prime}+1\right)$, i.e., $A\left(t^{\prime}+1\right)$ is to the right of and above $A\left(t^{\prime}\right)$. This implies that in time, provided that the system stays in Region A, in each period the outlets' audiences come closer to Region B, C, D or to the inner stationary state. Eventually, only four scenarios could occur:

1. For any $t>t^{\prime}$, the audiences remain in Region A. In this case, given the phase diagram, they necessarily converge to the inner stationary state.

2. At a certain $t^{\prime \prime}>t^{\prime}$, audiences jump to Region B. In this case, they will remain in Region $\mathrm{B}$ for any $t>t^{\prime \prime}$. First note that in this Region $A_{1}\left(t^{\prime \prime}\right)<A_{1}\left(t^{\prime \prime}+1\right)$ and $A_{2}\left(t^{\prime}\right)>A_{2}\left(t^{\prime \prime}+1\right)$, i.e., $A\left(t^{\prime \prime}+1\right)$ is to the right and below of $A\left(t^{\prime \prime}\right)$. Note that both $g_{1}\left(A_{1}\right)$ and $g_{2}\left(A_{1}\right)$ are strictly decreasing; thus, by Claims 2 and 3 , an outlet's audience can never jump out of this region for any $t>t^{\prime \prime}$. Consequently, in this case audiences necessarily converge to the inner stationary state.

3. At a certain $t^{\prime \prime}>t^{\prime}$, audiences jump to Region $\mathrm{C}$. This case is analogous to case 2; hence, audiences remain in Region $\mathrm{C}$ for any $t>t^{\prime \prime}$ and eventually converge to the inner stationary state.

\footnotetext{
30 Note that this does not imply that in the next period the dynamics will not jump over $g_{2}\left(A_{1}\right)$. It fact, this can occur because $A_{1}$ changes and $g_{2}\left(A_{1}\right)$ is decreasing.
} 
4. At a certain $t^{\prime \prime}>t^{\prime}$, audiences jump to Region D. In this case, the audiences at $t^{\prime \prime}-1$ are in Region $\mathrm{A}$, and at $t^{\prime \prime}$ they are in Region D. Let $A_{1}^{*}$ be the audience of outlet 1 in the inner stationary state. Necessarily $\left|A_{1}\left(t^{\prime \prime}-1\right)-A_{1}^{*}\right|>\left|A_{1}\left(t^{\prime \prime}\right)-A_{1}^{*}\right|$, because of Claims 2 and 3. In this region, the argument used in Region A can be applied. Therefore, audiences will converge to the inner stationary state given that if they jump from Region A to D and vice versa they will come closer to the inner stationary state in each period and will eventually converge.

The same logic applies to the study of the dynamics for an initial point in any of the other four regions.

Proof of Proposition $2 F_{1}\left(A_{1}, A_{2}\right)=-2 A_{1}^{3}-A_{1}^{2}\left(\alpha\left(1-\mu_{1}\right)^{2}+4 A_{2}-2\right)$ $-A_{1} A_{2}\left(\alpha\left(\left(\mu_{1}-4\right) \mu_{1}-\left(\mu_{2}-2\right) \mu_{2}+2\right)+2\left(\mu_{1}+A_{2}-2\right)\right)+2(\alpha-1) A_{2}^{2}\left(\mu_{1}-\mu_{2}\right)$. Operating,

$F_{2}\left(A_{1}, A_{2}\right)=-\left(2 A_{1}^{2}+2 A_{1}\left(-\alpha \mu_{2}+\alpha+\mu_{2}+2 A_{2}-2\right)+A_{2}\left(\alpha\left(\mu_{2}-1\right)^{2}+\right.\right.$ $\left.\left.2\left(A_{2}-1\right)\right)\right)$.

Setting $A_{1}=A_{2}=A$ and solving the equation system $\left\{F_{1}=0, F_{2}=0\right\}$ for $A$ and $\alpha$, we obtain that the solutions are $\alpha=-\frac{2}{\mu_{1}+\mu_{2}-4}$ and $A_{1}=A_{2}=\frac{1}{4} \frac{\left(\mu_{1}-3\right)\left(\mu_{2}-3\right)}{4-\left(\mu_{1}+\mu_{2}\right)}$. We define $\hat{\alpha}_{A}=-\frac{2}{\mu_{1}+\mu_{2}-4}$.

Proof of Proposition 3 Since $F_{1, \text { news }}=\mu_{2}-\mu_{1}+\frac{\left(1-\mu_{2}\right) A_{1}}{A_{1}+A_{2}}$ and $F_{2, \text { news }}=\frac{\left(1-\mu_{2}\right) A_{2}}{A_{1}+A_{2}}$, $F_{1, \text { news }} \leq F_{2, \text { news }}$ if and only if $A_{1} \leq A_{2} \frac{\left(1+\mu_{1}-2 \mu_{2}\right)}{1-\mu_{1}}$.

Next, we substitute $A_{1}=A_{2} \frac{\left(1+\mu_{1}-2 \mu_{2}\right)}{1-\mu_{1}}$ in the following two equations:

$F_{1}\left(A_{1}, A_{2}\right)=-2 A_{1}^{3}-A_{1}^{2}\left(\alpha\left(1-\mu_{1}\right)^{2}+4 A_{2}-2\right)-A_{1} A_{2}\left(\alpha\left(\left(\mu_{1}-4\right) \mu_{1}-\left(\mu_{2}-\right.\right.\right.$ 2) $\left.\left.\mu_{2}+2\right)+2\left(\mu_{1}+A_{2}-2\right)\right)+2(\alpha-1) A_{2}^{2}\left(\mu_{1}-\mu_{2}\right)=0$. Operating,

$F_{2}\left(A_{1}, A_{2}\right)=-\left(2 A_{1}^{2}+2 A_{1}\left(-\alpha \mu_{2}+\alpha+\mu_{2}+2 A_{2}-2\right)+A_{2}\left(\alpha\left(\mu_{2}-1\right)^{2}+\right.\right.$ $\left.\left.2\left(A_{2}-1\right)\right)\right)=0$.

Solving the system for $A_{2}$ and $\alpha$, we obtain $\alpha=\frac{4\left(1-\mu_{2}\right)}{5-\mu_{1}^{2}-6 \mu_{2}+2 \mu_{2} \mu_{1}}$, which is always greater than zero. However, it is smaller than one if and only if $\mu_{1}>2 \mu_{2}-1$.

\section{References}

Acemoglu D, Hassan T, Tahoun A (2017) The power of the street: evidence from Egypt's Arab spring. Rev Financ Stud 31:1-42

Adena M (2016) Nonprofit organizations, free media and donor's trust. J Econ 118(3):239-263

Allcott H, Gentzkow M (2017) Social media and fake news in the 2016 election. J Econ Perspect 31(2):211236

Anand B, Di Tella R, Galetovic A (2007) Information or opinion? Media bias as product differentiation. J Econ Manag Strategy 16(3):635-682

Anderson SP (2006) The media and advertising: a tale of two-sided markets. In: Ginsburgh V, Throsby D (eds) Handbook of the economics of art and culture, vol 1. Elsevier, Amsterdam, pp 567-614

Andina-Díaz A, García-Martínez JA (2018) Reputation and news suppression in the media industry. Working paper

Arifovic J, Curtis Eaton B, Walker G (2015) The coevolution of beliefs and networks. J Econ Behav Organ 120:46-63

Bakshy E, Messing S, Adamic LA (2015) Exposure to ideologically diverse news and opinion on facebook. Science 348(6239):1130-1132

Baron DP (2006) Persistent media bias. J Public Econ 90(1-2):1-36 
Besley T, Prat A (2006) Handcuffs for the grabbing hand? Media capture and government accountability. Am Econ Rev 96(3):720-736

Besley T, Burgess R (2001) Political agency, government responsiveness and the role of the media. Eur Econ Rev 45(4-6):629-640

Boxell L, Gentzkow M, Shapiro JM (2017) Is the internet causing political polarization? Evidence from demographics. Working paper

Cagé J (2014) Media competition, information provision and political participation. Working paper

Campante FR, Durante R, Sobbrio F (2018) Politics 2.0: the multifaceted effect of broadband internet on political participation. J Eur Econ Assoc 16(4):1094-1136

Casari M (2008) Markets in equilibrium with firms out of equilibrium: a simulation study. J Econ Behav Organ 65(2):261-276

Chen S-H, Huang Y-C (2008) Risk preference, forecasting accuracy and survival dynamics: simulations based on a multi-asset agent-based artificial stock market. J Econ Behav Organ 67(3):702-717

Djankov S, McLiesh C, Nenova T, Shleifer A (2003) Who owns the media? J Law Econ 46(2):341-381

Doyle G (2013) Understanding media economics. Sage Publications, Thousand Oaks

Egorov G, Guriev SM, Sonin K (2009) Why resource-poor dictators allow freer media: a theory and evidence from panel data. Am Polit Sci Rev 103(4):645-668

Ellman M, Germano F (2009) What do the papers sell? A model of advertising and media bias. Econ J 119(537):680-704

Enikolopov R, Makarin A, Petrova M (2016) Social media and protest participation: evidence from Russia. Working paper

Enikolopov R, Petrova M, Sonin K (2017) Socialmedia and corruption. Am Econ J 10:150-174

Gentzkow M, Shapiro JM (2006) Media bias and reputation. J Polit Econ 114(2):280-316

Gentzkow M, Shapiro JM (2011) Ideological segregation online and offline. Q J Econ 126(4):1799-1839

Gentzkow M, Shapiro JM, Sinkinson M (2014) Competition and ideological diversity: historical evidence from us newspapers. Am Econ Rev 104(10):3073-3114

Germano F, Sobbrio F (2017) Opinion dynamics via search engines (and other algorithmic gatekeepers). Working paper

Germano F, Meier M (2013) Concentration and self-censorship in commercial media. J Publ Econ 97:117_ 130

Groseclose T, Milyo J (2005) A measure of media bias. Q J Econ 120(4):1191-1237

Halberstam Y, Knight B (2016) Homophily, group size, and the diffusion of political information in social networks: evidence from twitter. J Publ Econ 143:73-88

Harrington JE (1999) Rigidity of social systems. J Polit Econ 107(1):40-64

Jackson MO, Rogers BW (2007) Relating network structure to diffusion properties through stochastic dominance. BE J Theor Econ 7(1):1-13

Kreindler GE, Young HP (2013) Fast convergence in evolutionary equilibrium selection. Games Econ Behav 80:39-67

Larcinese V, Puglisi R, Snyder JM (2011) Partisan bias in economic news: evidence on the agenda-setting behavior of U.S. newspapers. J Publ Econ 95(9):1178-1189

Lelarge M (2012) Diffusion and cascading behavior in random networks. Games Econ Behav 75(2):752-775

Little AT (2016) Communication technology and protest. J Politics 78(1):152-166

López-Pintado D (2006) Contagion and coordination in random networks. Int J Game Theory 34(3):371-381

López-Pintado D (2008) Diffusion in complex social networks. Games Econ Behav 62(2):573-590

Mitchell A, Weisel R (2014) Political polarization and media habits. Pew Research Center, Washington

Mullainathan S, Shleifer A (2005) The market for news. Am Econ Rev 95(4):1031-1053

Newman N (2017) Analysis by country: United Kingdom. Digital news report, Reuters Institute

Petrova M (2008) Inequality and media capture. J Publ Econ 92(1-2):183-212

Quattrociocchhi W, Caldarelli G, Scala A (2014) Opinion dynamics on interacting networks: media competition and social influence. Sci Rep 4:4938

Rochet J-C, Tirole J (2003) Platform competition in two-sided markets. J Eur Econ Assoc 1(4):990-1029

Strömberg D (2004a) Mass media competition, political competition, and public policy. Rev Econ Stud 71(1):265-284

Strömberg D (2004b) Radio's impact on public spending. Q J Econ 119(1):189-221

Vega-Redondo F, Marsili M, Slanina F (2005) Clustering, cooperation, and search in social networks. J Eur Econ Assoc 3(2-3):628-638 
Yang MJ, Chyi HI (2011) Competing with whom? Where? And why (not)? An empirical study of U.S. online newspapers' competition dynamics. J Media Bus Stud 8(4):59-74

Zaller J (1999) Market competition and news quality. Working paper

Publisher's Note Springer Nature remains neutral with regard to jurisdictional claims in published maps and institutional affiliations. 